\title{
10.
}

\section{Die allgemeinen unendlichen Reihen in der Analysis und ihre Darstellung in geschlossenen Ausdrücken.}

(Von Herrn J. Dienger, Lehrer der Mathematik und Physik an der höhern Bürgerschule zu Sinsheim bei Heidelberg.)

Die Analysis soll es ihrem Wesen nach nur mit continuirlichen Functionen zu thun haben. Sobald also eine von ihr behandelte Function aufhört continuirlich zu sein, hört auch der Gebrauch dieser Function für die Analysis auf, und fernere, etwa gültige Lehrsätze müssen auf anderem Wege abgeleitet werden. Kein Lehrsatz also, der, wenn die Bedingung auch nur stillschweigend gemacht wurde, die Continuität einer Function voraussetzt, darf auf diejenigen Fälle ausgedehnt werden, wo die Function aufhört, continuirlich zu sein; wenigstens könnte dies nur durch einen besondern, diesem Falle angepafsten Beweis geschehen. Dieses alleinige und ausschliefsliche Anwenden von continuirlichen Functionen liegt in der Natur der Mathematik überhaupt, und der Analysis insbesondere. Es kann daher auch die Frage, ob divergente Reihen gebraucht werden dürfen, gar nicht gestellt werden, und wenn sie etwa aufgestellt werden sollte, nur entschieden mit Nein beantwortet werden. Denn divergente Reihen und discontinuirliche Functionen sind gleichbedeutende Ausdrücke; und so wenig die Analysis berechtigt ist, ihre Lehrsätze auch auf den Fall auszudehnen, wo die Funclionen aufhören, continuirlich zu sein, ebensowenig dürfen ihre Lehrsätze die Fälle umfassen, wo etwa vorkommende Reihen aufhören convergent zu sein.

Es sind daher die Gesetze der Convergenz oder Divergenz unendlicher Reihen ein einzelner Fall der Gesetze der Continuität oder Discontinuität der Functionen überhaupt, und es würden jene unter diesen allgemeinern Gesetzen mitbegriffen sein.

In der vorliegenden Arbeit hat man speciell die in der Analysis gebräuchlichsten, unendlichen Reihen einer Prüfung ihrer Convergenz unterworfen, und dann gesucht, dieselben zu summiren, d. h. in geschlossenen Ausdrücken darzustellen. Es ist diese Aufgabe die umgekehrte der Entwicklung der Functionen in unendliche Reihen, scheint aber klarer und strenger zu sein, zumal in jedem Falle 
die Bedingungen, unter welchen das Resultat gültig oder ungültig ist, scharf und bestimmt hervortreten. Man hat dann aus den erhaltenen Resultaten andere herzuleiten gesucht; immer die Bedingungen der Gültigkeit oder Nichtgültigkeit im Auge behaltend.

Von den als bekannt angenommenen Sätzen findet sich bekanntlich eine umfassende Darstellung in Cauchy's Differentialrechnung, Vorlesung 11:; so wie auch in Ohms "Geist der math. Analysis", obwohl vielleicht manche Resultate noch einfacher herzuleiten wären.

Es sind verwandte Arbeiten benutzt worden; deren aber nicht viele vorhanden sind.

\section{\$. 1.}

Convergenz oder Divergenz unendlicher Reihen.

1. Eine beliebige Reihe

$$
\boldsymbol{k}_{0}+\boldsymbol{k}_{1}+\ldots+\boldsymbol{k}_{n}+\ldots
$$

möge convergent genannt werden, wenn für immer zunehmende Werthe von $n$ die Summe

$$
\boldsymbol{k}_{0}+\boldsymbol{k}_{1}+\ldots+\boldsymbol{k}_{n}
$$

sich beständig einer bestimmten, endlichen Grenze nähert: im entgegengesetzten Falle soll sie divergent heifsen. Die Grenze, wenn sie besteht, nennt man Grenze der Reihe.

2. Diese Erklärung vorausgesetzt, können folgende Lehrsälze leicht bewiesen werden (S. die Arbeit von Abel im 1ten Bande, und von Dr. Kumıner im 13ten Bande d. J.).

I. Lehrsatz. Bezeichnet man durch $\varrho_{0}, \varrho_{1}, \varrho_{2}, \ldots$ eine Reihe positiver Gröfsen, und năhert sich $\frac{\varrho_{n+1}}{\varrho_{n}}$, für stets wachsende Werthe von $n$, einer Gröfse $\alpha$, die gröfser ist als 1 , so wird die Reihe

$$
\varepsilon_{0} \varrho_{0}+\varepsilon_{1} \varrho_{1}+\varepsilon_{2} \varrho_{2}+\ldots .+\varepsilon_{n} \varrho_{n}+\ldots .
$$

wenn $\varepsilon_{n}$ eine Gröfse ist, die für stets wachsende Werthe von $n$ sich der Null nicht nähert, nothwendig divergiren.

II. Lehrsatz. Ist aber im I. Lehrsatze die Grōfse $\alpha$ kleiner als 1, so wird die Reihe

$$
\varepsilon_{0} \rho_{0}+\varepsilon_{1} \rho_{1}+\varepsilon_{2} \varrho_{2}+\ldots .+\varepsilon_{n} \rho_{n}+\ldots \ldots
$$

wenn $\varepsilon_{0}, \varepsilon_{1}, \ldots \varepsilon_{n}, \ldots$ die Einheit nicht ubersteigen, nothwendig convergiren. 
III. Lehrsatz. Wenn $v_{n}$ das allgemeine Glied einer Reihe ist, in welcher, von einem gewissen Gliede an, alle folgenden positive Vorzeichen haben, und man eine Function $m_{n}$, deren Werthe positiv sind, finden kann, von der Art, dafs $m_{n} v_{n}$ Null wird für $n=\infty$ und dafs

$$
\frac{m_{n} v_{n}}{v_{n+1}}-m_{n+1}
$$

grölser als Null ist für den nämlichen Werth von $n$, so ist die Reihe convergent.

Ist dagegen $\frac{m_{n} v_{n}}{v_{n+1}}-m_{n+1}$ gleich Null für $n=\infty$, so setze man

$$
f(n)=\frac{m_{n} v_{n}}{v_{n+1}}-m_{n+1}, \quad \varphi(n)=\frac{m_{n} v_{n}}{f(n)}
$$

und es ist zu diesem Lehrsatze noch Folgendes hinzuzufügen:

Ist für $n=\infty, f(n)$ Null und $\varphi(n)$ nicht Null, so ist die vorgelegte Reihe divergent.

IV. Lehrsatz. Bezeichnet man durch $\varrho_{0}, \varrho_{1}, \ldots, \varrho_{0}^{\prime}, \varrho_{1}^{\prime}, \rho_{2}^{\prime}, \ldots$ die Zahlenwerthe der Glieder zweier convergenter Reihen

$$
v_{0}+v_{1}+\ldots=p, \quad v_{0}^{\prime}+v_{1}^{\prime}+\ldots=p^{\prime}
$$

und sind die Reihen

$$
\varrho_{0}+\varrho_{1}+\ldots, \quad \varrho_{0}^{\prime}+\varrho_{1}^{\prime}+\ldots
$$

ebenfalls convergent, so wird auch die Reihe

$$
r_{0}+r_{1}+\ldots+r_{n}+\ldots
$$

deren allgemeines Glied

ist, convergent und ihre Summe gleich

$$
r_{n}=v_{0} v_{n}^{\prime}+v_{1} v_{n-1}^{\prime}+\ldots+v_{n} v_{0}^{\prime}
$$

$$
\left(v_{0}+v_{1}+\ldots\right)\left(v_{1}^{\prime}+v_{1}^{\prime}+\ldots\right)
$$

sein. Sind also die Reihen

$$
\begin{gathered}
t_{0}+t_{1}+t_{2}+\ldots \\
t_{0}^{\prime}+t_{1}^{\prime}+t_{2}^{\prime}+\ldots \\
t_{0} t_{0}^{\prime}+\left(t_{1} t_{0}^{\prime}+t_{1}^{\prime} t_{0}\right)+\left(t_{2} t_{0}+t_{1} t_{1}^{\prime}+t_{0} t_{2}^{\prime}\right)+\ldots
\end{gathered}
$$

convergent, so ist

$\left(t_{0}+t_{1}+t_{2}+\ldots\right)\left(t_{0}^{\prime}+t_{1}^{\prime}+t_{2}^{\prime}+\ldots\right)=t_{0} t_{0}^{\prime}+\left(t_{1} t_{0}^{\prime}+t_{0} t_{1}^{\prime}\right)+\left(t_{2} t_{0}^{\prime}+t_{1} t_{1}^{\prime}+t_{0} t_{2}^{\prime}\right)+\ldots$

§. 2 .

Bezeichnet man den Ausdruck

1. $1+a \frac{\dot{x}}{1}+a(a+k) \frac{x^{2}}{2 !}+a(a+k)(a+2 k) \frac{x^{3}}{3 !}+\ldots$, 
insofern er convergent (continuirlich) ist, durch $F(a)$, was auch $a, k, x$ sein mögen, so wird man den Ausdruck

$$
\text { 2. } \quad 1+b \frac{x}{1}+b(b+k) \frac{x^{2}}{2 !}+b(b+k)(b+2 k) \frac{x^{3}}{3 !}+\ldots
$$

ebenfalls, insofern er continuirlich (convergent) ist, durch $\boldsymbol{F}(\boldsymbol{b})$ zu bezeichnen haben. Es fragt sich nun, welche Form das Product $F(a) \cdot F(b)$ haben werde.

Multiplicirt man die beiden Reihen (1.) und (2.) wirklich, so findet man eine Reihe, die sich auf folgende Weise darstellen läfst:

$$
\text { 3. } 1+A_{1} x+A_{2} \frac{x^{2}}{2 !}+A_{3} \frac{x^{3}}{3 !}+\ldots+A_{n} \frac{x^{n}}{n !},
$$

und es ergiebt sich, dafs

$$
\begin{aligned}
& \boldsymbol{A}_{1}=a+b, \\
& \boldsymbol{A}_{2}=(a+b)(a+b+k), \\
& \boldsymbol{A}_{3}=(a+b)(a+b+k)(a+b+2 k) .
\end{aligned}
$$

Es findet also zwischen $\boldsymbol{A}_{1}, \boldsymbol{A}_{2}, \boldsymbol{A}_{3}$ folgender Zusammenhang Statt:

$$
\begin{aligned}
& \boldsymbol{A}_{2}=\boldsymbol{A}_{1}(\boldsymbol{a}+\boldsymbol{b}+\boldsymbol{k}), \\
& \boldsymbol{A}_{3}=\boldsymbol{A}_{2}(\boldsymbol{a}+\boldsymbol{b}+2 \boldsymbol{k}) .
\end{aligned}
$$

Sollte dies allgemein gelten, so müfste

$$
\boldsymbol{A}_{n}=\boldsymbol{A}_{n-1}(a+b+(n-1) k)
$$

sein. Nun ist

$$
\begin{aligned}
& \boldsymbol{A}_{n-1}=a(a+k)(a+2 k) \cdots(a+(n-2) k) \\
& +(n-1) b \cdot a(a+k) \cdots(a+(n-3) k) \\
& +\frac{n-1}{1} \cdot \frac{n-2}{2} \cdots \cdot \frac{n-r}{r} \cdot b(b+k) \cdots(b+(r-1) k) a(a+k) \cdots(a+(n-r-2) k) \\
& +b(b+k) \cdots(b+(n-2) k) \text { und } \\
& \boldsymbol{A}_{n}=a(a+k) \cdots(a+(n-1) k) \\
& +n b \cdot a(a+k) \cdots(a+(n-2) k) \\
& +\frac{n}{1} \cdot \frac{n-1}{2} \cdots \frac{n-r}{r+1} \cdot b(b+k) \cdots(b+r k) a(a+k) \cdots(a+(n-r-2) k) \\
& +b(b+k) \ldots(b+(n-1) k) \text {, }
\end{aligned}
$$

und bezeichnet man

$$
a(a+k) \cdots(a+r k)
$$

durch $\varphi(a, r)$, so ist, wie leicht zu sehen, 


$$
\begin{aligned}
& \boldsymbol{A}_{n-1}(a+b+(n-1) k)= \\
& \varphi(a, n-1)+b \varphi(a, n-2) \\
& +(n-1) b \varphi(a, n-1)+(n-1) \varphi(b, 1) \cdot \varphi(a, n-3) \\
& +\frac{n-1}{1} \cdot \frac{n-2}{2} \cdot \varphi(b, 1) \varphi(a, n-3)+\frac{n-1}{1} \cdot \frac{n-2}{2} \cdot \varphi(b, 2) \cdot \varphi(a, n-4) \\
& +\frac{n-1}{1} \cdot \frac{n-2}{2} \cdots \frac{n-r}{r} \cdot \varphi(b, r-1) \varphi(a, n-r-1) \\
& +\frac{n-1}{1} \cdot \frac{n-2}{2} \cdots \frac{n-r}{r} \cdot \varphi(b, r) \varphi(a, n-r-2) \\
& +\varphi(b, n-1) \text {. }
\end{aligned}
$$

Hieraus ergiebt sich, wenn man erwägt, dafs

$$
\begin{aligned}
n-1+1 & =n, \\
\frac{n-1}{1}+\frac{n-1}{1} \cdot \frac{n-2}{2} & =\frac{n}{1} \cdot \frac{n-1}{2}, \\
\frac{n-1}{1} \cdot \frac{n-2}{2}+\frac{n-1}{1} \cdot \frac{n-2}{2} \cdot \frac{n-3}{3} & =\frac{n}{1} \cdot \frac{n-1}{2} \cdot \frac{n-2}{3}
\end{aligned}
$$

u. s. f., wirklich

Also: wenn die Reihe

$$
A_{n-1}(a+b+(n-1) k)=A_{n}
$$

$1+(a+b) \frac{x}{1}+(a+b)(a+b+k) \frac{x^{2}}{2 !}+(a+b)(a+b+k)(a+b+2 k) \frac{x^{3}}{3 !}+\ldots$

convergent ist, in welchem Falle sie durch $\boldsymbol{F}(a+b)$ dargestellt wird, so ist

4. $\boldsymbol{F}(\boldsymbol{a}) \cdot \boldsymbol{F}(\boldsymbol{b})=\boldsymbol{F}(\boldsymbol{a}+\boldsymbol{b})$.

Da, unter der beständigen Voraussetzung, die angewandten und die gefundenen Reihen seien convergent (\$.1. Lehrsatz IV.),

$$
\boldsymbol{F}(\boldsymbol{a}) \cdot \boldsymbol{F}(\boldsymbol{b})=\boldsymbol{F}(\boldsymbol{a}+\boldsymbol{b})
$$

ist, so ist offenbar

$$
\boldsymbol{F}(\boldsymbol{a}) \cdot \boldsymbol{F}(\boldsymbol{b}+\boldsymbol{c})=\boldsymbol{F}(\boldsymbol{a}+\boldsymbol{b}+\boldsymbol{c})=\boldsymbol{F}(\boldsymbol{a}) \cdot \boldsymbol{F}(\boldsymbol{b}) \cdot \boldsymbol{F}(\boldsymbol{c}),
$$

und allgemein

$$
\boldsymbol{H}(\boldsymbol{a}) \cdot \boldsymbol{F}(\boldsymbol{b}) \cdot \boldsymbol{F}(\boldsymbol{c}) \ldots=\boldsymbol{F}(\boldsymbol{a}+\boldsymbol{b}+\boldsymbol{c}+\ldots)
$$

Setzt man $a=b=c \ldots$, und ist die Anzahl dieser Gröfsen $m$, so erhält man

$$
[\boldsymbol{F}(\boldsymbol{a})]^{m}=\boldsymbol{F}(\boldsymbol{m a} \boldsymbol{a})
$$

wenn $m$ eine ganze, positive Zahl ist.

Aus

$$
\boldsymbol{F}(\boldsymbol{a}) \cdot \boldsymbol{F}(\boldsymbol{b})=\boldsymbol{F}(\boldsymbol{a}+\boldsymbol{b})
$$

folgt 
und, wenn man $b-a$ stalt $b$ setzt,

$$
\boldsymbol{F}(\boldsymbol{b})=\frac{F(a+b)}{\boldsymbol{F}(\boldsymbol{a})},
$$

Aus $(\boldsymbol{F}(\boldsymbol{a}))^{m}=\boldsymbol{F}(\boldsymbol{m} \boldsymbol{a})$ erhält man

$$
\boldsymbol{F}(\boldsymbol{b}-\boldsymbol{a})=\frac{\boldsymbol{F}(\boldsymbol{b})}{\boldsymbol{F}(\boldsymbol{a})} .
$$

$$
\boldsymbol{F}(\boldsymbol{a})=\stackrel{m}{/}(\boldsymbol{F}(\boldsymbol{m} \boldsymbol{a}))
$$

und, wenn man $\frac{b}{m}$ statt $a$ setzt,

$$
\boldsymbol{F}\left(\frac{b}{m}\right)={ }^{m} /(\boldsymbol{F}(\boldsymbol{b}))=(\boldsymbol{F}(\boldsymbol{b}))^{\frac{1}{m}} .
$$

Hieraus, mit dem Obigen verbunden, ergiebt sich:

$$
\left(\boldsymbol{F}\left(\frac{b}{m}\right)\right)^{n}=\boldsymbol{F}\left(\frac{n b}{m}\right)=(\boldsymbol{F}(\boldsymbol{b}))^{\frac{n}{m}}
$$

Offenbar ist $\boldsymbol{F}(0)=1$, also

$$
(\boldsymbol{F}(a))^{-\frac{m}{r}}=\frac{1}{(F(a))^{\frac{m}{r}}}=\frac{F(0)}{(F(a))^{\frac{m}{r}}}=\frac{F(0)}{F\left(\frac{m a}{r}\right)}=\boldsymbol{F}\left(0-\frac{m a}{r}\right)=F\left(\frac{-m a}{r}\right) .
$$

Ist also $m$ eine reelle $Z a h l$, so ist

$$
\text { 5. }(\boldsymbol{F}(\boldsymbol{a}))^{m}=\boldsymbol{H}(\boldsymbol{m a}) \text {; }
$$

vorausgesetzt, dafs $\boldsymbol{F}(\boldsymbol{a}), \boldsymbol{F}(\boldsymbol{m a})$ convergente Reihen seien.

Setzt man $a=1, k=-1$, so giebt die Gleichung (5.):

6. $(1+x)^{m}=1+m x+\frac{m \cdot(m-1)}{1 \cdot 2} \cdot x^{2}+\frac{m \cdot(m-1)(m-2)}{1 \cdot 2 \cdot 3} \cdot x^{3}+\ldots$, vorausgesetzt, dafs die Reihe rechts convergent sei.

§. 3.

Bekanntlich läfst sich allgemein setzen:

$$
\alpha+\beta i=r(\cos \varphi+i \sin \varphi),
$$

wo $i=+1-1$ und

$$
r=+\sqrt{ }\left(\alpha^{2}+\beta^{2}\right), \quad \cos \varphi=\frac{\alpha}{r}, \quad \sin \varphi=\frac{\beta}{r}
$$

ist. Die Gröfse $\varphi$ werden wir in Bogentheilen (für den Radius 1) ausdrücken.

Ebenso hat man für ein ganzes positives $n$ :

$$
\begin{aligned}
& (\cos \varphi+i \sin \varphi)^{n}=\cos n \varphi+i \sin n \varphi, \\
& (\cos \varphi+i \sin \varphi)^{-n}=\cos n \varphi-i \sin n \varphi .
\end{aligned}
$$

Ist also

so ist

$$
\begin{gathered}
x=\alpha+\beta i=r(\cos \varphi+i \sin \varphi), \\
x^{n}=(\alpha+\beta i)^{n}=r^{n}(\cos n \varphi+i \sin n \varphi) .
\end{gathered}
$$


\$. 4.

Wir wollen nun zuerst die Reihe

1. $1+x+\frac{x^{2}}{2 !}+\frac{x^{3}}{3 !}+\ldots$

für $x=\alpha+\beta i=r(\cos \varphi+i \sin \varphi)$ betrachten.

Setzt man diesen Werth von $x$, so giebt die Reihe (1.)

2. $1+r \cos \varphi+\frac{r^{2}}{2 !} \cos 2 \varphi+\frac{r^{3}}{3 !} \cos 3 \varphi+\ldots$

$+i\left[r \sin \varphi+\frac{r^{2}}{2 !} \sin 2 \varphi+\frac{r^{3}}{3 !} \sin 3 \varphi+\ldots ..\right]$.

Untersuchen wir zuerst diese Reihe in Bezug auf ihre Convergenz oder Divergenz, so ist klar, dafs sie convergent oder divergent sein wird, je nachdem die Reihen

$$
\begin{gathered}
1+r \cos \varphi+\frac{r^{2}}{2 !} \cos 2 \varphi+\ldots, \\
r \sin \varphi+\frac{r^{2}}{2 !} \sin 2 \varphi+\ldots
\end{gathered}
$$

entweder beide convergent, oder beide (oder auch nur eine) divergent sind. Da $\cos n \varphi, \sin n \varphi$ sich der Null nicht nähern, die Einheit aber auch nicht übersteigen, so sind die beiden Reihen convergent und divergent, zugleich mit der Reihe

$$
1+r+\frac{r^{2}}{2 !}+\frac{r_{3}}{3 !}+\ldots
$$

(\$. 1. Lehrsatz I. II.). Für diese letztere Reihe ist aber $\varrho_{n}$ in $\$ .1:=\frac{r^{n}}{n !}$ also $\frac{\varrho_{n+1}}{\varrho_{n}}=\frac{r}{n+1}$, welche Gröfse für ein grofses $n$ immer kleiner als 1 ist, wenn $r$ nur irgend einen endlichen Werth erlangt. Die Reihe (2.) ist also convergent, und hat mithin eine Summe; welches auch der (endliche und bestimmle) Werth von $r$ und $\varphi$ sei. Setzt man

$$
\begin{array}{r}
1+r \cos \varphi+\frac{r^{2}}{2 !} \cos 2 \varphi+\frac{r^{3}}{3 !} \cos 3 \varphi+\ldots=\boldsymbol{P}, \\
r \sin \varphi+\frac{r^{2}}{2 !} \sin 2 \varphi+\frac{r^{3}}{3 !} \sin 3 \varphi+\ldots=\boldsymbol{Q},
\end{array}
$$

so ist die Summe der Reihe (1.) gleich

$$
\boldsymbol{P}+\boldsymbol{Q} \boldsymbol{i}
$$

und es wird sich blofs um die Bestimmung der Gröfsen $\boldsymbol{P}$ und $\boldsymbol{Q}$ handeln.

Die vorgelegte Reihe (1.) hat aber die Form der Reihe (1. \$. 2.), und sie entsteht aus ihr, wenn $\operatorname{man} k=0, x=1, a=x$ setzt: die vorliegende Reihe hat also auch die durch die Gleichung (4. \$. 2.) ausgedrückte characteristische Eigenschaft. 
Setzt man

$$
\boldsymbol{P}+\boldsymbol{Q} i=\boldsymbol{R}(\cos \boldsymbol{S}+i \sin \boldsymbol{S}),
$$

so wird man offenbar setzen dürfen:

$$
\boldsymbol{R}=\varphi(\alpha, \beta), \quad \boldsymbol{S}=\psi(\alpha, \beta) .
$$

Die Reihe (1.) ist demnach

$$
=\varphi(\alpha, \beta)[\cos \psi(\alpha, \beta)+i \sin \psi(\alpha, \beta)] \text {. }
$$

Selzt man in der Reihe (1.) $x=\alpha^{\prime}+\beta^{\prime} i$, so ist jene Reihe

$$
=\varphi\left(\alpha^{\prime}, \beta^{\prime}\right)\left[\cos \psi\left(\alpha^{\prime}, \beta^{\prime}\right)+i \sin \psi\left(\alpha^{\prime}, \beta^{\prime}\right)\right] .
$$

Das Product der beiden gefundenen Reihen läfst sich nach der Gleichung (4. \$. 2.) durch

$$
\varphi\left(\alpha+\alpha^{\prime}, \beta+\beta^{\prime}\right)\left[\cos \psi\left(\alpha+\alpha^{\prime}, \beta+\beta^{\prime}\right)+i \sin \psi\left(\alpha+\alpha^{\prime}, \beta+\beta^{\prime}\right)\right]
$$

ausdrücken und man erhält also

$$
\text { 3. } \begin{aligned}
& \varphi(\alpha, \beta) \cdot\left(\alpha^{\prime}, \beta^{\prime}\right)\left[\cos \left(\psi(\alpha, \beta)+\psi\left(\alpha^{\prime}, \beta^{\prime}\right)\right)+i \sin \left(\psi(\alpha, \beta)+\psi\left(\alpha^{\prime}, \beta^{\prime}\right)\right]\right. \\
= & \varphi\left(\alpha+\alpha^{\prime}, \beta+\beta^{\prime}\right)\left[\cos \psi\left(\alpha+\alpha^{\prime}, \beta+\beta^{\prime}\right)+i \sin \psi\left(\alpha+\alpha^{\prime}, \beta+\beta^{\prime}\right)\right],
\end{aligned}
$$

weil die Reihe (1.) immer convergent ist.

Aus dieser Gleichung (3.) ergeben sich bekanntlich folgende beiden:

4. $\left\{\begin{array}{l}\varphi\left(\alpha+\alpha^{\prime}, \beta+\beta^{\prime}\right) \cos \psi\left(\alpha+\alpha^{\prime}, \beta+\beta^{\prime}\right)=\varphi(\alpha, \beta) \cdot \varphi\left(\alpha^{\prime}, \beta^{\prime}\right) \cos \left[\psi(\alpha, \beta)+\psi\left(\alpha^{\prime}, \beta^{\prime}\right)\right], \\ \varphi\left(\alpha+\alpha^{\prime}, \beta+\beta^{\prime}\right) \sin \psi\left(\alpha+\alpha^{\prime}, \beta+\beta^{\prime}\right)=\varphi(\alpha, \beta) \cdot \varphi\left(\alpha^{\prime}, \beta^{\prime}\right) \sin \left[\psi(\alpha, \beta)+\psi\left(\alpha^{\prime}, \beta^{\prime}\right)\right] .\end{array}\right.$ Quadrirt man diese beiden Gleichungen, addirt, und bemerkt, dafs die durch $\varphi$ angedeuteten Functionen immer positiv sind, so erhält man

$$
\text { 5. }\left\{\begin{array}{l}
\varphi\left(\alpha+\alpha^{\prime}, \beta+\beta^{\prime}\right)=\varphi(\alpha, \beta) \varphi\left(\alpha^{\prime}, \beta^{\prime}\right), \\
\psi\left(\alpha+\alpha^{\prime}, \beta+\beta^{\prime}\right)=2 m \pi+\psi(\alpha, \beta)+\psi\left(\alpha^{\prime}, \beta^{\prime}\right),
\end{array}\right.
$$

wenn $m$ eine ganze Zahl ist. Diese Gleichungen (5.) sind zwei Bedingungsgleichungen, aus welchen die Gröfsen $\varphi$ und $\psi$ zu bestimmen sind.

Um dazu zu gelangen, kann man wie folgt verfahren:

Man nehme an, es sei eine Function $\psi(x)$ dergestalt zu bestimmen, dafs

$$
\text { 6. } \psi(x+y)=2 m \pi+\psi(x)+\psi(y) \text {. }
$$

Selzt man hier nach einander $y=x, 2 x, \ldots n x$ und substituirt die jeweils gefundenen Werthe, so erhält man:

$$
\text { 7. } \psi(n x)=n \psi(x)+(n-1) 2 m \pi \text {. }
$$

Setzt man in dieser Gleichung $x=\frac{z}{n}$, so erhält man

$$
\text { 8. }\left\{\begin{array}{l}
\psi(z)=n \psi\left(\frac{z}{n}\right)+(n-1) 2 m \pi, \\
\psi\left(\frac{z}{n}\right)=\frac{1}{n} \psi(z)+\left(\frac{1}{n}-1\right) 2 m \pi .
\end{array}\right.
$$


Aus dieser Gleichung, verbunden mit (7.), folgt:

$$
\text { 9. } \begin{aligned}
\psi\left(\frac{n x}{r}\right) & =n \psi\left(\frac{x}{r}\right)+(n-1) 2 m \pi \\
& =\frac{n}{r} \psi(x)+n\left(\frac{1}{r}-1\right) 2 m \pi+(n-1) 2 m \pi \\
& =\frac{n}{r} \psi(x)+\left(\frac{n}{r}-1\right) 2 m \pi .
\end{aligned}
$$

Für $x=0$ folgt aus (7.)

$$
\psi(0)=-2 m \pi \text {. }
$$

Setzt man also in (6.) $y=-x$, so findet sich

und daraus

$$
\psi(-x)=-\psi(x)-4 m \pi,
$$

$$
\begin{aligned}
\psi\left(-\frac{n x}{r}\right) & =-\psi\left(\frac{n x}{r}\right)-4 m \pi \\
& =-\frac{n}{r} \cdot \psi(x)+\left(-\frac{n}{r}-1\right) 2 m \pi
\end{aligned}
$$

Was also auch $n$ sei, so ist

$$
\psi(n x)=n \psi(x)+(n-1) 2 m \pi .
$$

Man selze nun $x=a, n=z$, so erhält man

Aber nach (7.) ist

$$
\psi(\boldsymbol{a} z)=(z-1) 2 m \pi+z \psi(a) .
$$

subtrahirt man diese beiden Gleichungen, so ergiebt sich

$$
\frac{z}{2 m \pi+\psi(z)}=\frac{a}{2 m \pi+\psi(a)}=\frac{1}{k} ;
$$

weil $\frac{a}{2 m \pi+\psi(a)}$ eine bestimmte Gröfse ist. Also erhält man

$$
\psi(x)=k x-2 m \pi ;
$$

wo $k$ eine bestimmte, unveränderliche Gröfse bezeichnet.

Die zweite Gleichung (5.) giebt für $\beta=\beta^{\prime}=\mathbf{0}$ :

$$
\psi\left(\alpha+\alpha^{\prime}, 0\right)=\psi(\alpha, 0)+\psi\left(\alpha^{\prime}, 0\right)+2 m \pi:
$$

eine Gleichung, die von der Form (6.) ist und aus welcher demnach

folgt. Desgleichen findet sich

$$
\psi(\alpha, 0)=k \alpha-2 m \pi
$$

$$
\psi(0, \beta)=k^{\prime} \beta-2 m \pi .
$$

Setzt man $\alpha=0, \beta^{\prime}=0$, so erhält man

$$
\begin{aligned}
\psi\left(\alpha^{\prime}, \beta\right) & =2 m \pi+\psi(0, \beta)+\psi\left(\alpha^{\prime}, 0\right) \\
& =2 m \pi+k^{\prime} \beta-2 m \pi+k \alpha^{\prime}-2 m \pi \\
& =k \alpha^{\prime}+k^{\prime} \beta-2 m \pi,
\end{aligned}
$$

Crelle's Journal f. d. M. Bd XXXIV. Heft 3. 
also

$$
\text { 10. } \psi(\alpha, \beta)=k \alpha+k^{\prime} \beta-2 m \pi
$$

wo $k$ und $k^{\prime}$ zwei bestimmte unveränderliche Zahlen sind.

Was $\varphi(\alpha, \beta)$ betrifft, so ist diese Gröfse immer positiv; also wird man setzen können:

$$
\varphi(\alpha, \beta)=e^{r(\alpha, \beta)} \text {. }
$$

Selzt man diesen Werth in die erste Gleichung (5.), und nimmt die Logarithmen, so erhält man

eine Gleichung, aus welcher wie oben

$$
\boldsymbol{F}\left(\alpha+\alpha^{\prime}, \beta+\beta^{\prime}\right)=\boldsymbol{F}(\alpha, \beta)+\boldsymbol{F}\left(\alpha^{\prime}, \beta^{\prime}\right):
$$

11. $\boldsymbol{F}(\alpha, \beta)=h \alpha+h^{\prime} \beta$

folgt; wo $h$ und $l^{\prime}$ bestimmte, unveränderliche Zahlen sind.

Demnach ist

12.

$$
\left\{\begin{aligned}
\boldsymbol{P}+\boldsymbol{Q} i & =\boldsymbol{e}^{h \alpha+h^{\prime} \beta}\left[\cos \left(\boldsymbol{k} \alpha+\boldsymbol{k}^{\prime} \beta\right)+i \sin \left(\boldsymbol{k} \alpha+\boldsymbol{k}^{\prime} \beta\right)\right] \\
\boldsymbol{P} & =\boldsymbol{e}^{h \alpha+h^{\prime} \beta} \cos \left(\boldsymbol{k} \alpha+\boldsymbol{k}^{\prime} \beta\right) \\
\boldsymbol{Q} & =\boldsymbol{e}^{h \alpha+h^{\prime} \beta} \sin \left(\boldsymbol{k} \alpha+\boldsymbol{k}^{\prime} \beta\right)
\end{aligned}\right.
$$

wo also blofs $h, h^{\prime}, k, k^{\prime}$ für beliebige Werthe van $r$ und $\varphi$ oder $\alpha$ und $\beta$ zu bestimmen sind, indem sie für alle solche Werthe sich nicht verändern.

Setzt man aber $\beta=0$, so ist $r=+\sqrt{ } \alpha^{2}, \cos \varphi=\frac{\alpha}{r}= \pm 1$, je nachdem $\alpha$ positiv oder negativ ist, und $\sin \varphi=0, \varphi=\left\{\begin{array}{l}0 \\ \pi\end{array}\right.$, je nachdem $\alpha$ positiv oder negativ ist. Also ergiebt sich dann

$$
\begin{aligned}
\boldsymbol{P} & =1 \pm r+\frac{r^{2}}{2 !} \pm \frac{r^{3}}{3 !}+\ldots \\
& =1+\alpha+\frac{\alpha^{2}}{2 !}+\frac{\alpha^{3}}{3 !}+\ldots
\end{aligned}
$$

Aber die Reihe (1. \$. 2.) wird für $a=1, k=0$ und $x=\alpha$ zu

$$
1+\alpha+\frac{\alpha^{2}}{2 !}+\frac{\alpha^{3}}{3 !}+\ldots
$$

Setzt man in der Formel (5. §. 2.) $m=\frac{1}{\alpha}, a=\alpha, k=0$ und $x=1$, so findet sich:

$$
\left(1+\alpha+\frac{\alpha^{2}}{2 !}+\frac{\alpha^{3}}{3 !}+\ldots\right)^{\frac{1}{\alpha}}=1+\frac{1}{1}+\frac{1}{2 !}+\frac{1}{3 !}+\frac{1}{4 !}+\ldots
$$

Da nun die Reihe

$$
1+\alpha+\frac{\alpha^{2}}{2 !}+\frac{\alpha^{3}}{3 !}+\ldots
$$


immer convergent ist, so hat auch

$$
1+1+\frac{1}{2 !}+\frac{1}{3 !}+\frac{1}{4 !}+\ldots
$$

einen bestimmten Werth $(e=2,7182818 \ldots)$; demnach ist

$$
1+\alpha+\frac{\alpha^{2}}{2 !}+\frac{\alpha^{3}}{3 !}+\ldots=e^{\alpha},
$$

indem bekanntlich die Grundzahl der natürlichen Logarithmen durch diese Formel ausgedrückt wird.

Also ist für $\beta=0$ :

$$
\boldsymbol{P}=\boldsymbol{e}^{\alpha}
$$

Aber aus (12.) folgt, für $\beta=0$ :

mithin ist

$$
\boldsymbol{P}=e^{h \alpha} \cos (k \alpha),
$$

und demnach

$$
e^{h \alpha} \cos k \alpha=e^{\alpha}
$$

$$
\text { 13. } h=1, \quad k=0 \text {. }
$$

Hieraus ergiebt sich nun, in Verbindung mit (12.) und den frühern Werthen von $\boldsymbol{P}$ und $\boldsymbol{Q}$ :

$$
\begin{aligned}
& e^{\alpha+h^{\prime} \beta} \cos k^{\prime} \beta=1+r \cos \varphi+\frac{r^{2}}{2 !} \cos 2 \varphi+\ldots \\
& e^{\alpha+h^{\prime} \beta} \sin k^{\prime} \beta=r \sin \alpha+\frac{r^{2}}{2 !} \sin 2 \alpha+\ldots
\end{aligned}
$$

Für $\alpha=0$ folgt leicht, wie oben:

$$
\begin{aligned}
& e^{h^{\prime} \beta} \cos k^{\prime} \beta=1-\frac{\beta^{2}}{2 !}+\frac{\beta^{4}}{4 !}-\frac{\beta^{6}}{6 !}+\ldots, \\
& e^{h^{\prime} \beta} \sin k^{\prime} \beta=\beta-\frac{\beta^{3}}{3 !}+\frac{\beta^{5}}{5 !}-\ldots
\end{aligned}
$$

und hieraus :

$$
\begin{aligned}
& e^{h^{\prime} \beta}\left(\cos k^{\prime} \beta+i \sin k^{\prime} \beta\right)=1+\beta i+\frac{(\beta i)^{2}}{2 !}+\frac{(\beta i)^{3}}{3 !}+\ldots \\
& e^{h^{\prime} \beta}\left(\cos k^{\prime} \beta-i \sin k^{\prime} \beta\right)=1-\beta i+\frac{(\beta i)^{2}}{2 !}-\frac{(\beta i)^{3}}{3 !}-\ldots
\end{aligned}
$$

Aber die beiden Reihen rechts haben die Form der Reihe (1. $\$$. 2.), wenn man $x=1, k=0, \alpha= \pm \beta i$ setzt; ihr Product ist also $\boldsymbol{F}(\beta i) \cdot \boldsymbol{F}(-\beta \boldsymbol{\beta} i)$ $=\boldsymbol{F}(\beta i-\beta i)=\boldsymbol{F}(0)=1$. Dies Product ist aber $e^{2 h^{\prime} \beta}$, also ist

$$
e^{2 h^{\prime} \beta}=1, \quad h^{\prime}=0,
$$


folglich

$$
\begin{aligned}
& \cos k^{\prime} \beta=1-\frac{\beta^{2}}{2 !}+\frac{\beta^{4}}{4 !}-\ldots, \\
& \sin k^{\prime} \beta=\beta-\frac{\beta^{3}}{3 !}+\frac{\beta^{3}}{5 !}-\ldots
\end{aligned}
$$

Nun nähert sich bekanntlich $\frac{\sin l^{\prime} \beta}{l^{\prime} \beta}$ für ein abnehmendes $\beta$ der Énheit; aber es ist

$$
\frac{\sin k^{\prime} \beta}{k^{\prime} \beta}=\frac{1}{k^{\prime}}\left(1-\frac{\beta^{2}}{3 !}+\frac{\beta^{4}}{5 !}-\ldots\right),
$$

welche Gröfse sich der Einheit nähert, wenn

$$
k^{\prime}=1
$$

mithin ist, nebenbei bemerkt, für jedes reelle $\beta$ :

$$
\text { 14. }\left\{\begin{array}{l}
\cos \beta=1-\frac{\beta^{2}}{2 !}+\frac{\beta^{4}}{4 !}-\ldots, \\
\sin \beta=\beta-\frac{\beta^{3}}{3 !}+\frac{\beta^{5}}{5 !}-\ldots .
\end{array}\right.
$$

Die Gleichungen (12.) geben also:

$$
\boldsymbol{P}=\boldsymbol{e}^{\alpha} \cos \beta, \quad \boldsymbol{Q}=\boldsymbol{e}^{\alpha} \sin \beta,
$$

und die Summe der vorgelegten Reihe ist

$$
e^{\alpha}(\cos \beta+i \sin \beta)
$$

Setzt man, nach der Analogie wie

$$
1+\alpha+\frac{\alpha^{2}}{2 !}+\ldots=e^{\alpha}
$$

auch

$$
1+(\alpha+\beta i)+\frac{(\alpha+\beta i)^{2}}{2 !}+\ldots=e^{u+i i}
$$

so ist

$$
\boldsymbol{e}^{\alpha+\beta i}=\boldsymbol{e}^{\alpha}(\cos \beta+i \sin \beta) \text {. }
$$

Zugleich ist, da $\boldsymbol{r}=+\sqrt{ }\left(\alpha^{2}+\beta^{2}\right), r \cos \varphi=\alpha, \boldsymbol{r} \sin \varphi=\beta, \boldsymbol{P}=e^{r \cos \varphi} \cos (\boldsymbol{r} \sin \varphi)$, $\boldsymbol{Q}=\boldsymbol{e}^{r \cos \varphi} \sin (\boldsymbol{r} \sin \varphi)$, wenn man die früher angegebenen Werthe von $\boldsymbol{P}$ und $\boldsymbol{Q}$ herücksichtigt:

$$
\text { 15. }\left\{\begin{aligned}
1+r \cos \varphi+\frac{r^{2}}{2 !} \cos 2 \varphi+\ldots \ldots & =e^{r \cos \varphi} \cos (r \sin \varphi), \\
r \sin \varphi+\frac{r^{2}}{2 !} \sin 2 \varphi+\ldots \ldots & =e^{r \cos \varphi} \sin (r \sin \varphi) .
\end{aligned}\right.
$$

Diese beiden Reihen gelten, was auch immer die Werthe von $r$ und $\varphi$ sein mögen. 
\$. 5.

Solchergestalt gefundene Reihen lassen mannigfache Umbildungen zu. Zuerst wollen wir in den Reihen (15. \$. 4.) $\pi-\varphi$ statt $\varphi$ setzen. Dies giebt, allgemein gültig:

1. $\left\{\begin{array}{r}1-r \cos \varphi+\frac{r^{2}}{2 !} \cos 2 \varphi-\frac{r^{3}}{3 !} \cos 3 \varphi+\ldots=e^{-r \cos \varphi} \cos (r \sin \varphi), \\ r \sin \varphi-\frac{r^{2}}{2 !} \sin 2 \varphi+\frac{r^{3}}{3 !} \sin 3 \varphi-\ldots=e^{-r \cos \varphi} \sin (r \sin \varphi) .\end{array}\right.$

Addirt man diese Gleichungen und diejenigen (15. \$. 4.), so findet sich

2. $\left\{\begin{aligned} 1+\frac{r^{2}}{2 !} \cos 2 \varphi+\frac{r^{4}}{4 !} \cos 4 \varphi+\ldots & =\frac{1}{2}\left(e^{r \cos \varphi}+e^{-r \cos \varphi}\right) \cos (r \sin \varphi), \\ r \sin \varphi+\frac{r^{3}}{3 !} \sin 3 \varphi+\ldots & =\frac{1}{2}\left(e^{r \cos \varphi}+e^{-r \cos \varphi}\right) \sin (r \sin \varphi) ;\end{aligned}\right.$

desgleichen durch Subtraction:

3. $\left\{\begin{aligned} r \cos \varphi+\frac{r^{3}}{3 !} \cos 3 \varphi+\frac{r^{5}}{5 !} \cos 5 \varphi+\ldots & =\frac{1}{2}\left(e^{r \cos \varphi}-e^{-r \cos \varphi}\right) \cos (r \sin \varphi), \\ \frac{r^{2}}{2 !} \sin 2 \varphi+\frac{r^{4}}{4 !} \sin 4 \varphi+\ldots & =\frac{1}{2}\left(e^{r \cos \varphi}-e^{-r \cos \varphi}\right) \sin (r \sin \varphi) .\end{aligned}\right.$

Ähnliche Reihen findet man, wenn man $\frac{1}{2} \pi-\varphi$ statt $\varphi$ setzt; nur ist ihr Fortschreitungsgesetz nicht mehr so einfach. Diese Reihen sind:

4. $\left\{\begin{array}{r}1+r \sin \varphi-\frac{r^{2}}{2 !} \cos 2 \varphi-\frac{r^{3}}{3 !} \sin 3 \varphi+\frac{r^{4}}{4 !} \cos 4 \varphi+\ldots=e^{r \sin \varphi} \cos (r \cos \varphi), \\ r \cos \varphi+\frac{r^{2}}{2 !} \sin 2 \varphi-\frac{r^{3}}{3 !} \cos 3 \varphi-\frac{r^{4}}{4 !} \sin 4 \varphi+\ldots=e^{r \sin \varphi} \sin (r \cos \varphi) .\end{array}\right.$

Diese einfachen Umbildungen sind aber nicht die einzigen, welche abgeleitet werden können. Eine ganze Reihe von Formeln kann durch Differenziirung und Integrirung gefunden werden. Es ist nämlich das Differenzial oder Integral einer Reihe dem Differenzial oder Integral des sie darstellenden geschlossenen Ausdrucks gleich, wenn die neue Reihe convergent ist.

Man kann nun nach $\boldsymbol{r}$ oder $\varphi$ differenziiren oder integriren, und es ist dadurch der Bildung neuer Formeln ein weites Feld geöffnet. Betrachten wir in dieser Beziehung zuerst die erste der Gleichungen (15. \$. 4.). Differenziirt man nach $r$, so giebt die erste Seite:

$\cos \varphi+r \cos 2 \varphi+\frac{r^{2}}{2 !} \cos 3 \varphi+\frac{r^{3}}{3 !} \cos 4 \varphi+\ldots$;

welche Reihe immer convergent ist. Überhaupt ist klar, dafs alle Differenzialquotienten dieser Reihe convergent sein werden. Der Differenzialquotient 
von der Ordnung $k$ ist

$$
\cos k \varphi+r \cos (k+1) \varphi+\frac{r^{2}}{2 !} \cos (k+2) \varphi+\frac{r^{3}}{3 !} \cos (k+3) \varphi+\ldots
$$

Der Differenzialquotient von der nämlichen Ordnung für die zweite Seite ist für $y=p \cdot q$, wenn $\boldsymbol{p}$ und $\boldsymbol{q}$ zwei Functionen der Veränderlichen $\boldsymbol{r}$ sind:

$$
\frac{d^{k} y}{d r^{k}}=p \cdot \frac{d^{k} q}{d r^{k}}+k \cdot \frac{d p}{d r} \cdot \frac{d^{k-1} q}{d r^{k-1}}+\frac{k \cdot k-1}{1 \cdot 2} \cdot \frac{d^{2} p}{d r^{2}} \cdot \frac{d^{k-2} q}{d r^{k-2}}+\ldots+\frac{d^{k} p}{d r^{k}} \cdot q .
$$

Setzt $\operatorname{man} p=e^{r \cos \varphi}, q=\cos (r \sin \varphi)$, so ist

$$
\frac{d^{k} p}{d r^{k}}=\cos ^{k} \varphi e^{r \cos \varphi}, \quad \frac{d^{k} q}{d r^{k}}=\sin ^{k} \varphi \cdot \cos \left(\frac{1}{2} k \pi+r \sin \varphi\right) ;
$$

demnach ist

$$
\frac{d^{k} e^{r \cos \varphi} \cos (r \sin \varphi)}{d r^{k}}
$$

$=e^{r \cos \varphi} \sin ^{k} \varphi \cdot \cos \left(\frac{1}{2} k \pi+r \sin \varphi\right)+k \cos \varphi \cdot \sin ^{2} \varphi e^{r \cos \varphi} \cos \left(\frac{1}{2}(k-1) \pi+r \sin \varphi\right)+\ldots$. $=e^{r \cos \varphi}\left[\cos ^{k} \varphi \cdot \cos (r \sin \varphi)-k \cos ^{k-1} \varphi \cdot \sin \varphi \cdot \sin (r \sin \varphi-\ldots]\right.$.

Das allgemeine Glied dieser Reihe ist

$$
\frac{k \cdot(k-1) \cdots(k-v+1)}{1 \cdot 2 \cdots \cdot v} \cdot e^{r \cos \varphi} \cdot \cos ^{v} \varphi \cdot \sin ^{k-v} \varphi \cdot \cos \left(\frac{1}{2}(k-v) \pi+r \sin \varphi\right),
$$

wenn man $\frac{k(k-1) \cdots(k-v+1)}{1 \cdot 2 \cdots v}$ für $v=0$ der Einheit gleichsetzt. Es ist demnach

$$
\begin{gathered}
\frac{d^{k} e^{r \cos \varphi} \cos (r \sin \varphi)}{d r^{k}} \\
=\sum_{v=0}^{v=k} \frac{k \cdot(k-1) \cdots(k-v+1)}{v !} \cdot e^{r \cos \varphi} \cos ^{v} \varphi \cdot \sin ^{k-v} \varphi \cdot \cos \left[\frac{1}{2}(k-v) \pi+r \sin \varphi\right]
\end{gathered}
$$

und folglich

5. $\cos k \varphi+r \cos (k+1) \varphi+\frac{r^{2}}{2 !} \cos (k+2) \varphi+\frac{r^{3}}{3 !} \cos (k+3) \varphi+\ldots$

$$
\begin{aligned}
& =\sum_{v=0}^{v=k} \frac{k \cdot(k-1) \cdots(k-v+1)}{v !} \cdot e^{r \cos \varphi} \cos ^{v} \varphi \cdot \sin ^{k-v} \varphi \cdot \cos \left[\frac{1}{2}(k-v) \pi+r \sin \varphi\right] \\
& =\frac{d^{k} \cdot e^{r \cos \varphi} \cos (r \sin \varphi)}{d r^{k}}
\end{aligned}
$$

was auch $k, \varphi, r$ sein mögen.

Aus der zweiten Reihe (15. \$.4.) erhält man ganz eben so:

6. $\sin k \varphi+r \sin (k+1) \varphi+\frac{r^{2}}{2 !} \sin (k+2) \varphi+\frac{r^{3}}{3 !} \sin (k+3) \varphi+\ldots$

$=\sum_{v=1}^{v=k} \frac{k \cdot(k-1) \cdots \cdot(k-v+1)}{v !} e^{r \cos \varphi} \cos ^{v} \varphi \cdot \sin ^{k-v} \varphi \cdot \sin \left[\frac{1}{2}(k-v) \pi+r \sin \varphi\right]$

$=\frac{d^{k} e^{r \cos \varphi} \sin (r \sin \varphi)}{d r^{k}}$. 
Setzt man in diesen beiden letzten Reihen $\pi-\varphi$ statt $\varphi$, und bemerkt, dafs $\sin (k \pi-k \varphi)=(-1)^{k+1} \sin k \varphi, \cos (k \pi-k \varphi)=(-1)^{k} \cos k \varphi$, so findet sich

7. $\cos k \varphi-r \cos (k+1) \varphi+\frac{r^{2}}{2 !} \cos (k+2) \varphi-\frac{r^{3}}{3 !} \cos (k+3) \varphi+\ldots$

$=\sum_{v=0}^{v=k} \frac{k \cdot(k-1) \cdots \cdot(k-v+1)}{v !} \cdot e^{-r \cos \varphi} \cos ^{v} \varphi \cdot \sin ^{k-v} \varphi \cdot \cos \left[\frac{1}{2}(k-v) \pi+r \sin \varphi\right] \cdot(-1)^{k+1}$

$=(-1)^{k} \frac{d^{k} e^{-r \cos \varphi} \cos (r \sin \varphi)}{d r^{k}}$ und

8. $\quad \sin k \varphi-r \sin (k+1) \varphi+\frac{r^{2}}{2 !} \sin (k+2) \varphi+\frac{r^{3}}{3 !} \sin (k+3) \varphi-\ldots$

$=\sum_{v=1)}^{v=k} \frac{k \cdot(k-1) \cdots \cdot(k-v+1)}{v !} \cdot e^{-r \cos \varphi} \cos ^{v} \varphi \cdot \sin ^{k-v} \varphi \cdot \sin \left[\frac{1}{2}(k-v) \pi+r \cdot \sin \varphi\right](-1)^{k+1+v}$

$=(-1)^{k+1} \frac{d^{k} e^{-r \cos \varphi} \sin (r \sin \varphi)}{d r^{k}}$.

Durch Addition der Gleichungen (5. und 7. und 6. und 8.) erhält man

9. $\quad \cos k \varphi+\frac{r^{2}}{2 !} \cos (k+2) \varphi+\frac{r^{4}}{4 !} \cos (k+4) \varphi+\ldots$

$$
=\frac{\frac{1}{2} d^{k}\left[\left(e^{r \cos \varphi}+(-1)^{k} e^{-r \cos \varphi}\right) \cos (r \sin \varphi)\right]}{d r^{k}} \text { und }
$$

10. $\sin k \varphi+\frac{r^{2}}{2 !} \sin (k+2) \varphi+\frac{r^{4}}{4 !} \sin (k+4) \varphi+\ldots$

$$
=\frac{\frac{1}{2} d^{k}\left[\left(e^{r \cos \varphi}+(-1)^{k+1} e^{-r \cos \varphi}\right) \sin (r \sin \varphi)\right]}{d r^{k}} \text {. }
$$

\$. 6 .

Eine Reihe von Formeln erhält man, wenn man nach $\varphi$ differenziirt. Da sich aber die Differenzialquotienten der zweiten Seiten nicht so einfach ausdrücken lassen, so wollen wir blofs eine einzige Formel ableiten. Differenziirt man die Formeln (15.) einmal, so ergiebt sich

$$
\begin{aligned}
& r \sin \varphi+r^{2} \sin 2 \varphi+\frac{r^{3}}{2 !} \sin 3 \varphi+\frac{r^{4}}{3 !} \sin 4 \varphi+\ldots \\
& =e^{r \cos \varphi} r[\sin \varphi \cdot \cos (r \sin \varphi)+\cos \varphi \cdot \sin (r \sin \varphi)] \\
& =r e^{r \cos \varphi} \sin (\varphi+r \sin \varphi)
\end{aligned}
$$

oder

1. $\sin \varphi+r \sin 2 \varphi+\frac{r^{2}}{2 !} \sin 3 \varphi+\ldots=e^{r \cos \varphi} \sin (\varphi+r \sin \varphi)$; $r \cos \varphi+r^{2} \cos 2 \varphi+\frac{r^{3}}{2 !} \cos 3 \varphi+\frac{r^{4}}{3 !} \cos 4 \varphi+\ldots$ $=e^{r \cos \varphi} r(\cos (r \sin \varphi) \cos \varphi-\sin (r \sin \varphi) \sin \varphi)=r e^{r \cos \varphi} \cos (\varphi+r \sin \varphi)$, 
also

2. $\cos \varphi+r \cos 2 \varphi+\frac{r^{2}}{2 !} \cos 3 \varphi+\frac{r^{3}}{3 !} \cos 4 \varphi+\ldots=e^{r \cos \varphi} \cos (\varphi+r \sin \varphi)$.

Setzt man in diesen letzten Reihen $\pi-\varphi$ statt $\varphi$, so erhält man:

3.

$$
\left\{\begin{aligned}
\sin \varphi-r \sin 2 \varphi+\frac{r^{2}}{2 !} \sin 3 \varphi-\frac{r^{3}}{3 !} \sin 4 \varphi+\ldots & =+e^{-r \cos \varphi} \sin (\pi-\varphi+r \sin \varphi) \\
& =-e^{-r \cos \varphi} \sin (r \sin \varphi-\varphi) \\
\cos \varphi-r \cos 2 \varphi+\frac{r^{2}}{2 !} \cos 3 \varphi-\frac{r^{3}}{3 !} \cos 4 \varphi+\ldots . & =-e^{-r \cos \varphi} \cos (\pi-\varphi+r \sin \varphi) \\
& =+e^{-r \cos \varphi} \cos (r \sin \varphi-\varphi)
\end{aligned}\right.
$$

\section{\$. 7.}

Da

$$
\begin{aligned}
\int e^{r \cos \varphi} \cos (\boldsymbol{r} \sin \varphi) d \boldsymbol{r} & =\frac{\cos \varphi \cdot \cos (r \sin \varphi)+\sin \varphi \cdot \sin (r \sin \varphi)}{\cos ^{2} \varphi+\sin ^{2} \varphi} \cdot e^{r \cos \varphi} \\
& =\cos (\boldsymbol{r} \sin \varphi-\varphi) e^{r \cos \varphi},
\end{aligned}
$$

so ist, wenn man die erste Gleichung (15.) nach $\boldsymbol{r}$ integrirt:

$$
r+\frac{r^{2}}{2 !} \cos \varphi+\frac{r^{3}}{3 !} \cos 2 \varphi+\ldots+\text { Const. }=\cos (r \sin \varphi-\varphi) e^{r \cos \varphi} .
$$

Für $\varphi=0$ findet man

$$
\boldsymbol{r}+\frac{r^{2}}{2 !}+\frac{r^{3}}{3 !}+\ldots+C=e^{r},
$$

also $C=1$ und mithin

1. $1+r+\frac{r^{2}}{2 !} \cos \varphi+\frac{r^{3}}{3 !} \cos 2 \varphi+\ldots=\cos (r \sin \varphi-\varphi) e^{r \cos \varphi}$.

Diese Gleichung läfst wieder Integrationen zu, allein das Gesetz des Fortschreitens ist nicht einfach. Es folgt zugleich, dafs die Gröfse

$$
\cos (r \sin \varphi-\varphi) e^{r \cos \varphi}-1
$$

sich durch $r$ theilen lasse und dafs

$$
\frac{\cos (r \sin \varphi-\varphi) e^{r \cos \varphi}-1}{r}
$$

gleich 1 sei für $r=0$.

Eben so hat man

$$
\begin{aligned}
\int e^{r \cos \varphi} \sin (r \sin \varphi) d r & =\frac{\cos \varphi \cdot \sin (r \sin \varphi)-\sin \varphi \cdot \cos (r \sin \varphi)}{\sin ^{2} \varphi+\cos ^{2} \varphi} \cdot e^{r \cos \varphi} \\
& =\sin (r \sin \varphi-\varphi) e^{r \cos \varphi}
\end{aligned}
$$

also

$$
C+\frac{r^{2}}{2 !} \sin \varphi+\frac{r^{3}}{3 !} \sin 2 \varphi+\frac{r^{\cdot 4}}{3 !} \sin 3 \varphi+\ldots=\sin (r \sin \varphi-\varphi) e^{r \cos \varphi} .
$$


Für $\varphi=0$ ist ebenfalls $C=0$, also

2. $\quad \frac{r^{2}}{2 !} \sin \varphi+\frac{r^{3}}{3 !} \sin 2 \varphi+\frac{r^{4}}{4 !} \sin 3 \varphi+\ldots=\sin (r \sin \varphi-\varphi) e^{r \cos \varphi}$.

Daraus folgt, dafs sich

$$
\sin (\boldsymbol{r} \sin \varphi-\varphi) e^{r \cos \varphi}
$$

durch $r^{2}$ theilen lasse und dafs der Quotient $=\frac{1}{2} \sin \varphi$ sei für $r=0$.

\$. 8 .

Neue Formeln ergeben sich, wenn man den Gröfsen $\boldsymbol{r}$ und $\varphi$ in den vorigen Paragraphen besondere Werthe beilegt. Es sei $r=+1$. Dann erhält man

$$
\begin{aligned}
& 1+\cos \varphi+\frac{1}{2 !} \cos 2 \varphi+\frac{1}{3 !} \cos 3 \varphi+\ldots=+e^{+\cos \varphi} \cos (\sin \varphi), \\
& +\sin \varphi+\frac{1}{2 !} \sin 2 \varphi+\frac{1}{3 !} \sin 3 \varphi+\ldots=+e^{+\cos \varphi} \sin (\sin \varphi) \\
& 1-\cos \varphi+\frac{1}{2 !} \cos 2 \varphi-\frac{1}{3 !} \cos 3 \varphi+\ldots=+e^{-\cos \varphi} \cos (\sin \varphi), \\
& +\sin \varphi-\frac{1}{2 !} \sin 2 \varphi+\frac{1}{3 !} \sin 3 \varphi-\ldots=+e^{-\cos \varphi} \sin (\sin \varphi), \\
& 1+\frac{1}{2 !} \cos 2 \varphi+\frac{1}{4 !} \cos 4 \varphi+\ldots=\frac{1}{2}\left(e^{\cos \varphi}+e^{-\cos \varphi}\right) \cos (\sin \varphi), \\
& \quad \sin \varphi+\frac{1}{3 !} \sin 3 \varphi+\ldots=\frac{1}{2}\left(e^{\cos \varphi}+e^{-\cos \varphi}\right) \sin (\sin \varphi), \\
& \sin \varphi+\frac{1}{1} \sin 2 \varphi+\frac{1}{2 !} \sin 3 \varphi+\frac{1}{3 !} \sin 4 \varphi+\ldots=e^{+\cos \varphi} \sin (\varphi+\sin \varphi), \\
& \cos \varphi+\frac{1}{1} \cos 2 \varphi+\frac{1}{2 !} \cos 3 \varphi+\frac{1}{3 !} \cos 4 \varphi+\ldots=e^{+\cos \varphi} \cos (\varphi+\sin \varphi), \\
& \sin \varphi-\sin 2 \varphi+\frac{1}{2 !} \sin 3 \varphi-\ldots=-e^{-\cos \varphi} \sin (\sin \varphi-\varphi), \\
& \cos \varphi-\cos 2 \varphi+\frac{1}{2 !} \cos 3 \varphi-\ldots=+e^{-\cos \varphi} \cos (\sin \varphi-\varphi) .
\end{aligned}
$$

Da sich weitere Formeln aus den ersten nicht auf einfachem Wege ergeben, so wenden wir uns zur Binomialreihe.

§. 9 .

Die Reihe

$$
\text { 1. } 1+m x+\frac{m \cdot(m-1)}{1 \cdot 2} \cdot x^{2}+\frac{m \cdot(m-1)(m-2)}{1 \cdot 2 \cdot 3} \cdot x^{3}+\ldots .
$$

nemlich die Binomialreihe, ist ebenfalls von der Form der Reihe (1. \$. 2.). Setzt man dort $a=m, K=-1$, so erhält man sie. Bezeichnet man also, im Fall 
die Reihe (1.) convergent ist, ihre Summe durch $\varphi(m)$, so ist

$$
\text { 2. } \varphi(\boldsymbol{m}) \cdot \varphi(\boldsymbol{n})=\varphi(\boldsymbol{m}+\boldsymbol{n}) \text {. }
$$

Es sei nun $x=a+b i=r(\cos \alpha+i \sin \alpha), \frac{m-v+1}{v}=d_{v} \cos \left(e_{v}+i \sin e_{v}\right)$, so ist $r=+\sqrt{ }\left(a^{2}+b^{2}\right), \cos \alpha=\frac{a}{r}, \sin \alpha=\frac{b}{r}, d_{v}=+\sqrt{ }\left(\left(\frac{p-v+1}{v}\right)^{2}+\left(\frac{q}{v}\right)^{2}\right)$, $\cos e_{\nu}=\frac{p-v+1}{v d_{v}}, \quad \sin e_{\nu}=\frac{q}{v d_{v}}$, wenn $m=p+q i$.

Setzt man, um abzukürzen,

$$
d_{1} d_{2} \ldots d_{v}=D_{v}, \quad e_{1}+e_{2}+\ldots+e_{v}+v \alpha=E_{v},
$$

so ist die Reihe (1.) folgende:

$$
\text { 3. } \begin{array}{cc} 
& \left.\boldsymbol{1}+\boldsymbol{D}_{1} \cos \boldsymbol{E}_{1} \boldsymbol{r}+\boldsymbol{D}_{2} \cos \boldsymbol{E}_{2} \boldsymbol{r}^{2}+\ldots\right) \\
& +\boldsymbol{i}\left(\boldsymbol{D}_{1} \sin \boldsymbol{E}_{1} \cdot \boldsymbol{r}+\boldsymbol{D}_{2} \sin \boldsymbol{E}_{2} \cdot \boldsymbol{r}^{2}+\ldots\right) .
\end{array}
$$

Es ist also diese Reihe in Bezug auf Convergenz oder Divergenz zu untersuchen.

Da $\cos \boldsymbol{E}_{n}$ und $\sin \boldsymbol{E}_{n}$ die Einheit nicht übersteigen, so ist die Reihe zugleich mit

$$
1+\boldsymbol{D}_{1} \boldsymbol{r}+\boldsymbol{r}^{2} \boldsymbol{D}_{2}+\boldsymbol{r}^{3} \boldsymbol{D}_{3}+\ldots
$$

convergent. Die Gröfse $\varrho_{n}$ in (\$. 1.) ist also

$$
=\boldsymbol{r}^{n} \boldsymbol{D}_{n}
$$

und folglich

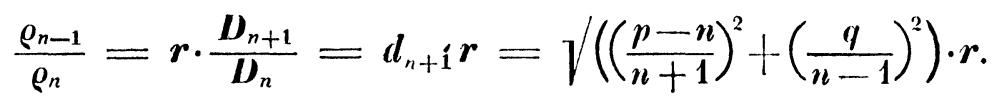

Für ein unendliches $n$ ist diese Gröfse $=r$.

Die vorgelegte Reihe also ist convergent für $r<1$ und divergent für $r>1$. Setzt man demnach in der Reihe (6. $\$$. 2.) $m$ und $x$ reell, so hat jene Gleichung Statt, wenn $x^{2}<1$ ist.

Es bleibt noch der Fall zu untersuchen, wenn $r=1$ ist.

In diesem Fall ist die Reihe convergent und divergent zugleich mit

$$
\boldsymbol{1}+\boldsymbol{D}_{\mathbf{1}}+\boldsymbol{D}_{2}+\boldsymbol{D}_{3}+\ldots
$$

Hiebei sind drei Fälle zu unterscheiden:

1ter Fall. $p=-1$ oder $<-1$. In diesem Falle kann man $\boldsymbol{p}=-1-n$ und $\boldsymbol{n} \equiv 0$ setzen; also ist dann

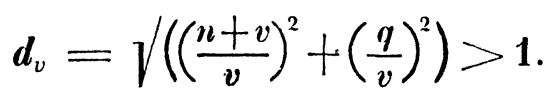

Aber da $\boldsymbol{D}_{v}=d_{1} d_{2} \ldots d_{v}$ ist, so ist $\boldsymbol{D}_{v}>1$, und $\boldsymbol{D}_{v}$ wird sich mit wachsendem Werthe von $v$ der Null nicht nähern; die Reihe wird also in diesem Falle divergent sein. 
2ter Fall. $p>0$ oder positiv.

Es sei $c$ eine positive Zahl und $<p$, so ist

$$
\begin{aligned}
& (v-p-1+c)^{2}=(v-p-1)^{2}+2 c(v-p-1)+c^{2}, \text { also } \\
& (v-p-1)^{2}+q^{2}=(v-p-1+c)^{2}-2 c(v-p-1)-c^{2}+q^{2} .
\end{aligned}
$$

Setzt man nun

$$
v>p+1+\frac{q^{2}}{2 c}-\frac{1}{2} c,
$$

so ist offenbar $q^{2}-2 c(v-p-1)-c^{2}<0$, also alsdann

$$
\left(\frac{v-p-1}{v}\right)^{2}+\frac{q^{2}}{v^{2}}<\left(\frac{v-p-1+c}{v}\right)^{2},
$$

folglich, dem Zahlenwerthe nach,

$$
d_{v}<\frac{v-p-1+c}{v} \text { oder }<1-\frac{p+1-c}{v} .
$$

Nun ist aber für ein reelles $n(6 . \$$. 2.)

$$
\left(1+\frac{1}{v}\right)^{-n}=1-\frac{n}{v}+\frac{n \cdot(n+1)}{1 \cdot 2}\left(\frac{1}{v^{2}}-\frac{n+2}{3 v} \cdot \frac{1}{v^{2}}\right)+\ldots
$$

Setzt man also $n=1+p-c$, so ist $v>n$ und also

$$
\left(1+\frac{1}{v}\right)^{-1-p+c}>1-\frac{1+p-c}{v},
$$

mithin

$$
d_{v}<\left(\frac{v+1}{v}\right)^{-1-p+c} \text { oder }<\left(\frac{v}{v+1}\right)^{1+p-c}
$$

Da $v>p+1-\frac{1}{2} c+\frac{q^{2}}{2 c}$ ist, so setze man

$$
p+1-\frac{1}{2} c+\frac{q^{2}}{2 c}=r
$$

wenn diese Gröfse eine ganze Zahl ist; oder es sei $r$ die ganze Zahl, die unmittelbar gröfser ist als $p+1-\frac{1}{2} c+\frac{q^{2}}{2 c}$. Es sei nun $v=r+n$, so folgt für $\boldsymbol{r}>\boldsymbol{n}$ :

$$
d_{r+n}<\left(\frac{r+n}{r+n+1}\right)^{1+p-c}
$$

Setzt man hier nach einander $n=1,2,3, \ldots m$ und multiplicirt die Resultate, so erhält man

$$
\begin{gathered}
d_{r+1} d_{r+2} \ldots d_{r+m}<\left(\frac{r+1}{r+m+1}\right)^{1+p-c} \text { oder } \\
D_{r+m}<d_{1} d_{2} \ldots d_{r}\left(\frac{r+1}{r+m+1}\right)^{1+p-c} .
\end{gathered}
$$


Setzt man hierin $m=1,2, \ldots n$ und addirt, während $\operatorname{man} D_{r}$ auf beiden Seiten beifügt, so erhält man:

$$
\begin{gathered}
D_{r}+D_{r+1}+\ldots+D_{r+n} \\
<d_{1} d_{2} \ldots d_{r}\left(\frac{1}{(r+1)^{1+p-c}}+\frac{1}{(r+2)^{1+p-c}}+\ldots+\frac{1}{(r+n+1)^{1+p-c}}\right)(r+1)^{1+p-c} .
\end{gathered}
$$

Nun ist

also

$$
\left(1-\frac{1}{r+s+1}\right)^{c-p}=1+\frac{p-c}{r+s+1}+\frac{(p-c)(p-c+1)}{1 \cdot 2(r+s+1)^{2}}+\ldots,
$$

$$
\left(\frac{r+s}{r+s+1}\right)^{c-p}>1+\frac{p-c}{r+s+1} \text { oder } \frac{p-c}{r+s+1}<\left(\frac{r+s+1}{r+s}\right)^{p-c}-1 .
$$

Dividirl man beiderseits mit $(\boldsymbol{p}-\boldsymbol{c})(\boldsymbol{r}+\boldsymbol{s}+1)^{p-c}$, so erhält man

$$
\frac{1}{(r+s+1)^{1+p-c}}<\frac{1}{p-c}\left(\frac{1}{(r+s)^{p-c}}-\frac{1}{(r+s+1)^{p-c}}\right) \text {. }
$$

Setzt man hier nach einander $s=0,1, \ldots n$, und addirt, so findet sich

$$
\begin{aligned}
& \frac{1}{(r+1)^{1+p-c}}+\frac{1}{(r+1)^{1+p-c}}+\cdots+\frac{1}{(r+n+1)^{p-1+1}} \\
< & \frac{1}{p-c}\left(\frac{1}{r^{p-c}}-\frac{1}{(r+n+1)^{p-c}}\right) \text { oder }<\frac{1}{p-c} \cdot \frac{1}{r^{p-c}}:
\end{aligned}
$$

Also ist immer

$$
\begin{aligned}
& \boldsymbol{D}_{r}+\boldsymbol{D}_{r+1}+\ldots+\boldsymbol{D}_{r+n}<\boldsymbol{d}_{1} \boldsymbol{d}_{2} \ldots \boldsymbol{d}_{r} \cdot \frac{(\boldsymbol{r}+1)^{1+p-c}}{(p-c) r^{p-c}} \text { und } \\
& \boldsymbol{D}_{1}+\boldsymbol{D}_{2}+\ldots+\boldsymbol{D}_{n}<\boldsymbol{D}_{1}+\boldsymbol{D}_{2}+\ldots \ldots+\boldsymbol{D}_{r}+\boldsymbol{d}_{1} \boldsymbol{d}_{2} \ldots \boldsymbol{d}_{r} \cdot \frac{(\boldsymbol{r}+1)^{1+p-c}}{(p-1) r^{p-c}}
\end{aligned}
$$

was auch $n$ sei. Die Summe der Reihe

$$
\boldsymbol{D}_{1}+\boldsymbol{D}_{2}+\boldsymbol{D}_{3}+\ldots
$$

bleibt mithin immer endlich und bestimmt, und die Reihe demnach convergent.

3ter Fall. Es sei $p=0$, oder auch $p<0$ und $>-1$.

Da $m=p+q i, x=\cos \alpha+i \sin \alpha$ ist, so setze man

$$
\frac{m \cdot(m-1) \cdot(m-2) \ldots(m-n+1)}{n !}=m_{n},
$$

so dafs die Reihe

$$
1+m_{1} x+m_{2} x^{2}+\ldots .+m_{n} x^{n} \text { ist. }
$$

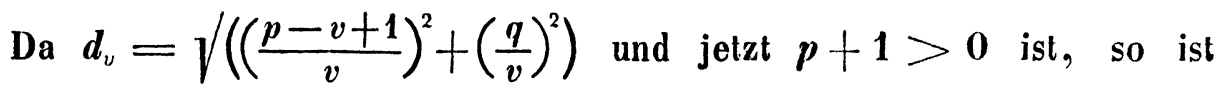

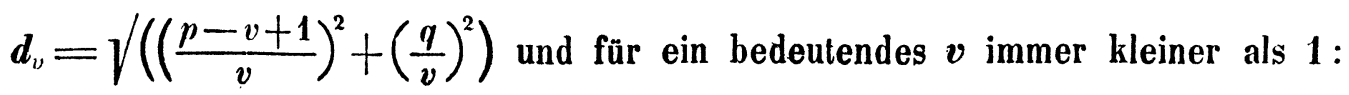
also wird $D_{v}=d_{1} d_{2} \ldots d_{v}$ 
für ein sehr grofses $v$ verschwinden; folglich auch $m_{\nu}$ für ein solches $v$. Setzt man nun

$$
1+m_{1} x+m_{2} x^{2}+\ldots+m_{v} x^{v}=\boldsymbol{P}_{v}
$$

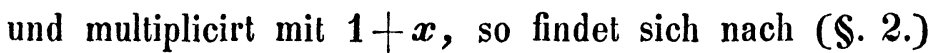

$$
1+(m+1)_{1} x+(m+1)_{2} x^{2}+\ldots+(m+1)_{v} x^{v}=P_{v}(1+x)-m_{v} x^{2+1} \text {. }
$$

Setzt man $v$ unendlich grofs, so ist die Reihe links convergent, da $m+1>0$ (2ter Fall); $\boldsymbol{n}_{v}$ ist $=0$; also ist, wenn $\boldsymbol{K}$ die Summe der Reihe links bezeichnet, für ein unendliches $v$ :

$$
\boldsymbol{K}=\boldsymbol{P}_{v}(1+x) \quad \text { und } \quad \boldsymbol{P}_{v}=\frac{\boldsymbol{K}}{1+\boldsymbol{x}} .
$$

Ist demnach $1+x$ nicht $=0$, so hat die Reihe in diesem Falle auch eine bestimmte Summe $=\frac{K}{1+x}$ und ist folglich convergent. Ist aber

so ist sie divergent.

$$
1+x=0 \quad \text { oder } \quad \cos \alpha=-1
$$

Fassen wir das Gesagte zusammen, so ergiebt sich, dafs die Reihe (1.) convergent ist:

1) Für jeden Werth von $p$ und $q$, wenn $r$ oder $l^{\prime}\left(a^{2}+b^{2}\right)<1$.

2) Für $\sqrt{ }\left(a^{2}+b^{2}\right)=1$ ist sie convergent, wenn $p>-1$, falls nicht zugleich $a=-1$ ist, in welchem letzteren Falle $p>0$ sein muls.

In jedem andern Falle ist die Reihe divergent.

Sucht man nun für die Fälle, in welchen die vorgelegte Reihe convergent ist, ihre Summe, so findet sich leicht, vermöge der Gleichung (2.) und des im \$. 4. Gesagten, dafs die Summe

$$
=e^{\delta p+\varepsilon q}(\cos (\beta p+\gamma q)+i \sin (\beta p+\gamma q))
$$

ist, wenn $\delta, \varepsilon, \beta, \gamma$ bestimmte, nicht mit $p$ und $q$ veränderliche Zahlen sind. Setzt man also die Summe der Reihe $=$

so ist

$$
\boldsymbol{P}+\boldsymbol{Q} i,
$$

Für $q=0$ ist

$$
\text { 4. }\left\{\begin{array}{l}
\boldsymbol{P}=e^{\delta p+\varepsilon q} \cos (\beta \boldsymbol{p}+\gamma \boldsymbol{q}), \\
\boldsymbol{Q}=\boldsymbol{e}^{\delta p+\varepsilon q} \sin (\beta \boldsymbol{p}+\gamma \boldsymbol{q}) .
\end{array}\right.
$$

$$
4^{\prime} . \quad\left\{\begin{array}{l}
\boldsymbol{P}=e^{\delta p} \cos \beta p=1+p \cos \alpha \cdot r+\frac{p \cdot(p-1)}{1 \cdot 2} \cos 2 \alpha \cdot r^{2}+\ldots \\
\boldsymbol{Q}=e^{\delta p} \sin \beta p=p \sin \alpha \cdot r+\frac{p \cdot(p-1)}{1 \cdot 2} \sin 2 \alpha \cdot r^{2}+\ldots
\end{array}\right.
$$


Diese Reihen gelten jedenfalls für $p=1$, wenn auch $r=1$ ist. Für $p=1$ erhält man daraus

und hieraus

$$
e^{\delta} \cos \beta=1+r \cos \alpha, \quad e^{\delta} \sin \beta=r \sin \alpha,
$$

$$
\text { 5. }\left\{\begin{array}{l}
\beta=\operatorname{arc}\left(\operatorname{tang}=\frac{r \sin \alpha}{1+r \cos \alpha}\right)+n \pi, \\
\delta=l \cdot\left(1+2 r \cos \alpha+r^{2}\right)^{\frac{1}{2}},
\end{array}\right.
$$

wenn man durch $\operatorname{arc}\left(\operatorname{tang}=\frac{r \sin \alpha}{1+r \cos \alpha}\right)$ den (numerisch) kleinsten Bogen bezeichnet, dessen Tangente $\frac{r \sin \alpha}{1+r \cos \alpha}$ ist. $n$ ist eine noch zu bestimmende ganze Zahl.

Setzt man $p=0$, so ist $d_{1}=D_{1}=+\sqrt{ } q^{2}, \cos e_{1}=0, \sin e_{1}=1$, wenn man $q$ positiv annimmt, also $e_{1}=\frac{1}{2} \pi$; ferner $\cos e_{v}=\frac{-v+1}{v d v}, \sin e_{v}=\frac{q}{v d v}$, $d_{v}=\sqrt{ }\left(\left(\frac{v-1}{v}\right)^{2}+\left(\frac{q}{v}\right)^{2}\right)$. Demnach ist $D_{v}=d_{1} d_{2} \ldots d_{v}$ durch $q$ theilbar und $\boldsymbol{E}_{v}=e_{1}+e_{2} \ldots+e_{v}+v \alpha=\frac{1}{2} \pi+\boldsymbol{E}_{v}^{\prime}$, wenn $\boldsymbol{E}_{v}^{\prime}=\boldsymbol{e}_{2}+\ldots+\boldsymbol{e}_{0}+\boldsymbol{v} \alpha$; also $\cos \boldsymbol{E}_{v}=-\sin \boldsymbol{E}_{v}^{\prime}$ und $\sin \boldsymbol{E}_{v}=\cos \boldsymbol{E}_{v}^{\prime}$; also ist in diesem Falle:

$$
\text { 6. }\left\{\begin{array}{l}
\boldsymbol{P}=1-\boldsymbol{q} \sin \alpha \cdot \boldsymbol{r}-\boldsymbol{D}_{2} \sin \boldsymbol{E}_{2}^{\prime} \boldsymbol{r}^{2}-\ldots=e^{\varepsilon q} \cos \gamma \boldsymbol{q}, \\
\boldsymbol{Q}=\boldsymbol{q} \cos \alpha \cdot \boldsymbol{r}+\boldsymbol{D}_{2} \cos \boldsymbol{E}_{2}^{\prime} \boldsymbol{r}^{2}+\ldots=e^{\varepsilon q} \sin \gamma \boldsymbol{q} ;
\end{array}\right.
$$

für $r<1$, und für $r=1$, wenn $\cos \alpha$ nicht $=-1$.

Hieraus folgt unter, den nämlichen Bedingungen:

$$
\text { 7. }\left\{\begin{array}{l}
\frac{e^{\varepsilon q} \cos (\gamma q)-1}{q}=-\sin \alpha \cdot \boldsymbol{r}-\frac{\boldsymbol{D}_{2}}{\boldsymbol{q}} \sin \boldsymbol{E}_{2}^{\prime} \cdot \boldsymbol{r}^{2}-\ldots, \\
\frac{\boldsymbol{e}^{\varepsilon q} \sin (\gamma q)}{\boldsymbol{q}}=+\boldsymbol{r} \cos \alpha+\frac{\boldsymbol{D}_{2}}{\boldsymbol{q}} \cos \boldsymbol{E}_{2}^{\prime} \cdot \boldsymbol{r}^{2}+\ldots
\end{array}\right.
$$

Setzt man in die erste Gleichung für $e^{\varepsilon q}$ seinen Werth aus (\$.4.), in die zweite für $\sin \gamma q$ seinen Werth aus (14. \$. 4.) und hierauf $q=0$, so wird $e_{v}=\pi$, $\boldsymbol{E}_{v}^{\prime}=(v-1) \pi+v \alpha$ und demzufolge aus den Gleichungen:

$$
\text { 8. }\left\{\begin{array}{l}
\varepsilon=-r \sin \alpha+\frac{1}{2} r^{2} \sin 2 \alpha-\frac{1}{3} r^{3} \sin 3 \alpha+\ldots, \\
\gamma=+r \cos \alpha-\frac{1}{2} r^{2} \cos 2 \alpha+\frac{1}{3} r^{3} \cos 3 \alpha-\ldots ;
\end{array}\right.
$$

unter den gleichen Bedingungen, wie bei (6.).

Ebenso erhält man aus den Gleichungen $\left(4^{\prime}\right.$.) für $p=0$ :

$$
8^{\prime} .\left\{\begin{array}{l}
\delta=r \cos \alpha-\frac{1}{2} r^{2} \cos 2 \alpha+\frac{1}{3} r^{3} \cos 3 \alpha-\ldots, \\
\beta=r \sin \alpha-\frac{1}{2} r^{2} \sin 2 \alpha+\frac{1}{3} r^{3} \sin 3 \alpha-\ldots,
\end{array}\right.
$$

also

$$
\text { 9. } \gamma=\delta, \quad \varepsilon=-\beta \text {. }
$$

Da für $r=0$ auch $\beta=0$ ist, so mufs im Werthe von $\beta, n=0$ sein. 
Die Summe der Reihe (1.), in den Fällen wo sie convergent ist, ist also

$$
\begin{array}{r}
e^{p l \cdot\left(1+2 r \cos a+r^{2}\right)^{\frac{1}{2}}-q \operatorname{arc}\left(\operatorname{tang}=\frac{r \sin \alpha}{1+r \cos \alpha}\right)}\left(\cos \left[p \operatorname{arc}\left(\operatorname{tang}=\frac{r \sin \alpha}{1+r \cos \alpha}\right)+q l\left(1+2 r \cos \alpha+r^{2}\right)^{\frac{1}{2}}\right]\right. \\
\left.+i \sin \left[p \cdot \operatorname{arc}\left(\operatorname{tang}=\frac{r \sin \alpha}{1+r \cos \alpha}\right)+q l \cdot\left(1+2 r \cos \alpha+r^{2}\right)^{\frac{1}{2}}\right]\right) \\
=\left((1+a)^{2}+b^{2}\right)^{\frac{1}{2} p} \cdot e^{-q \operatorname{arc}\left(\tan \alpha=\frac{b}{1+a}\right)}\left[\cos \left(p \cdot \operatorname{arc}\left(\operatorname{tang}=\frac{b}{1+a}\right)+\frac{1}{2} q l\left((1+a)^{2}+b^{2}\right)\right)\right. \\
\left.+i \sin \left(p \cdot \operatorname{arc}\left(\operatorname{tang}=\frac{b}{1+a}\right)+\frac{1}{2} q l\left((1+a)^{2}+b^{2}\right)\right)\right] .
\end{array}
$$

Dieser letzte Ausdruck ist aber der einfachste Werth von

$$
(1+\boldsymbol{a}+\boldsymbol{b} \boldsymbol{i})^{p+q i}
$$

dem also die vorliegende Reihe gleichzusetzen ist, falls sie convergirt.

§. 10.

Aus den Resultaten in (\$. 9.) lassen sich nun eine Reihe von Formeln entwickeln.

Aus den Formeln $\left(8^{\prime}\right.$.) folgt, wenn man in diesem Paragraph immer $r$ positiv setzt:

1. $\left\{\begin{array}{l}r \cos \alpha-\frac{1}{2} r^{2} \cos 2 \alpha+\frac{1}{3} r^{3} \cos 3 \alpha-\ldots=\frac{1}{2} l\left(1+2 r \cos \alpha+r^{2}\right), \\ r \sin \alpha-\frac{1}{2} r^{2} \sin 2 \alpha+\frac{1}{3} r^{3} \sin 3 \alpha-\ldots=\operatorname{arc}\left(\operatorname{tang}=\frac{r \sin \alpha}{1+r \cos \alpha}\right),\end{array}\right.$

für $r<1$. Den Bogen nimmt man seinem kleinsten numerischen Werthe nach.

2. $\left\{\begin{aligned} \cos \alpha-\frac{1}{2} \cos 2 \alpha+\frac{1}{3} \cos 3 \alpha-\ldots & =\frac{1}{2} l(2+2 \cos \alpha) \\ & =l \cdot\left(2 \cos \frac{1}{2} \alpha\right), \\ \sin \alpha-\frac{1}{2} \sin 2 \alpha+\frac{1}{3} \sin 3 \alpha-\ldots . & =\operatorname{arc}\left(\operatorname{tang}=\frac{\sin \alpha}{1+\cos \alpha}\right)=\frac{1}{2} \alpha ;\end{aligned}\right.$

ausgenommen, wenn $\alpha=\pi$, oder in der ersten Formel $\cos \alpha=-1$ ist. Setzt man in den Formeln (1.) und (2.) $\pi-\alpha$ statt $\alpha$, so erhält man:

3. $\quad\left\{\begin{array}{l}r \cos \alpha+\frac{1}{2} r^{2} \cos 2 \alpha+\frac{1}{3} r^{3} \cos 3 \alpha+\ldots=-\frac{1}{2} l\left(1-2 r \cos \alpha+r^{2}\right), \\ r \sin \alpha+\frac{1}{2} r^{2} \sin 2 \alpha+\frac{1}{3} r^{3} \sin 3 \alpha+\ldots=\operatorname{arc}\left(\operatorname{tang}=\frac{r \sin \alpha}{1-r \cos \alpha}\right),\end{array}\right.$ für $r<1$;

$$
\text { 4. }\left\{\begin{array}{l}
\cos \alpha+\frac{1}{2} \cos 2 \alpha+\frac{1}{3} \cos 3 \alpha+\ldots=-l \cdot\left(2 \sin \frac{1}{2} \alpha\right), \\
\sin \alpha+\frac{1}{2} \sin 2 \alpha+\frac{1}{3} \sin 3 \alpha+\ldots=\frac{1}{2}(\pi-\alpha)
\end{array}\right.
$$

ausgenommen für $\cos \alpha=+1$, also gültig für $\alpha>0$ und $<2 \pi$, dieses ausschliefslich. Ist $\alpha=2 \pi+k$, so ist

$$
\sin \alpha+\frac{1}{2} \sin 2 \alpha+\frac{1}{3} \sin 3 \alpha+\ldots=\frac{1}{2}(\pi-k) \text {. }
$$


Aus den Formeln (2.) und (4.) folgt

ferner, dafs die Reihe

$$
\text { 5. }\left\{\begin{array}{l}
1-\frac{1}{2}+\frac{1}{3}-\frac{1}{4}+\ldots=l \cdot(2) \text { und } \\
1-\frac{1}{3}+\frac{1}{5}-\frac{1}{4}+\ldots=\frac{1}{4} \pi
\end{array}\right.
$$

divergent ist.

$$
1+\frac{1}{2}+\frac{1}{3}+\frac{1}{4}+\ldots
$$

Da $e^{\delta p}=\left(1+2 r \cos \alpha+r^{2}\right)^{\frac{k p}{p}}, \cos \beta p=\cos \operatorname{arc}\left(\operatorname{tang}=\frac{r \sin \alpha}{1+r \cos \alpha}\right) p$ ist, so folgt aus (4'. \$.9.):

$$
\text { 6. }\left\{\begin{array}{c}
1+p \cos \alpha \cdot r+\frac{p \cdot(p-1)}{1 \cdot 2} \cos 2 \alpha \cdot r^{2}+\ldots \\
=\left(1+2 r \cos +r^{2}\right)^{\frac{2}{2} p} \cos \operatorname{arc}\left(\tan g=\frac{r \sin \alpha}{1+r \cos \alpha}\right) p, \\
p \sin \alpha \cdot r+\frac{p \cdot(p-1)}{1 \cdot 2} \sin 2 \alpha \cdot r^{2}+\ldots \\
=\left(1+2 r \cos +r^{2}\right)^{\frac{1}{2} p} \sin \operatorname{arc}\left(\operatorname{tang}=\frac{r \sin \alpha}{1+r \cos \alpha}\right) p,
\end{array}\right.
$$

für $r<1$. Setzt man $r=+1$, so erhält man:

7. $\left\{\begin{array}{r}1+p \cos \alpha+\frac{p \cdot(p-1)}{1 \cdot 2} \cos 2 \alpha+\ldots=2^{p} \cos ^{p} \frac{1}{2} \alpha \cos \frac{1}{2} p \alpha, \\ p \sin \alpha+\frac{p \cdot(p-1)}{1 \cdot 2} \sin 2 \alpha+\ldots=2^{p} \cos ^{p} \frac{1}{2} \alpha \sin \frac{1}{2} p \alpha,\end{array}\right.$ wenn $p>-1$ und nicht $\cos \alpha=-1$; ist aber $\cos \alpha=-1$, so mufs $p>0$ sein.

Setzt man in den Formeln (6. und 7.) $\pi-\alpha$ statt $\alpha$, so findet sich

für $r<1$ und

$$
\text { 8. }\left\{\begin{array}{c}
1-p \cos \alpha \cdot r+\frac{p \cdot(p-1)}{1 \cdot 2} \cos 2 \alpha \cdot r^{2}-\ldots \\
\left(1-2 r \cos \alpha+r^{2}\right)^{\frac{1}{2} p} \cos \operatorname{arc}\left(\operatorname{tang}=\frac{r \sin \alpha}{1-r \cos \alpha}\right) p, \\
p \sin \alpha \cdot r-\frac{p \cdot(p-1)}{1 \cdot 2} \sin 2 \alpha \cdot r^{2}+\ldots \\
=\left(1-2 r \cos \alpha+r^{2}\right)^{\frac{1}{2} p} \cos \operatorname{arc}\left(\operatorname{tang}=\frac{r \sin \alpha}{1-r \cos \alpha}\right) p,
\end{array}\right.
$$

9. $\left\{\begin{array}{r}1-p \cos \alpha+\frac{p \cdot(p-1)}{1 \cdot 2} \cos 2 \alpha-\ldots=2^{p} \sin ^{p} \frac{1}{2} \alpha \cos \frac{1}{2} p(\pi-\alpha), \\ p \sin \alpha-\frac{p \cdot(p-1)}{1 \cdot 2} \sin 2 \alpha+\ldots=2^{p} \sin ^{p} \frac{1}{2} \alpha \sin \frac{1}{2} p(\pi-\alpha),\end{array}\right.$ für $p>-1$, wenn nicht $\cos \alpha=+1$; ist aber $\cos \alpha=+1$, so mufs $p>0$ sein. 
Also ist

für $p>0$, und

$$
\text { 10. } 1-p+\frac{p \cdot(p-1)}{1 \cdot 2}-\ldots=0
$$

$$
\text { 11. } 1+p+\frac{p \cdot(p-1)}{1 \cdot 2}+\ldots=2^{p}
$$

für $p>-1$.

Da die vorliegenden Formeln verwickelt sind, so ist es nicht wahrscheinlich, dafs durch Differenziirung oder Integrirung einfache Resultate zu erzielen wären.

\section{§. 11.}

Wir sahen in \$. 9., dafs, wenn der Modul $r$ von $x$ kleiner ist als die Einheit,

$$
(1+x)^{m}=1+m x^{m-1}+\frac{m \cdot(m-1)}{1 \cdot 2} \cdot x^{m-2}+\ldots
$$

ist. Man setze nun

$$
x=\frac{1}{a+b i}=\frac{a-b i}{a^{2}+b^{2}}=\frac{1}{r}(\cos \alpha-i \sin \alpha),
$$

so erhält man

$$
\text { 1. }\left\{\begin{aligned}
\left(1+\frac{1}{a+b i}\right)^{m} & =1+m \cdot\left(\frac{1}{a+b i}\right)^{m-1}+\frac{m \cdot(m-1)}{1 \cdot 2} \cdot\left(\frac{1}{a+b i}\right)^{m-2}+\ldots \ldots, \\
\left(\frac{1}{a+b i}\right)^{m}(1+a+b i)^{m} & =1+m \cdot\left(\frac{1}{a+b i}\right)^{m-1}+\frac{m \cdot(m-1)}{1 \cdot 2} \cdot\left(\frac{1}{a+b i}\right)^{m-2}+\ldots \ldots
\end{aligned}\right.
$$

welche Formel für $r>1$ gilt.

Nimmt man $m$ imaginär, $=p+q i$ und $a+b i=r(\cos \alpha+i \sin \alpha)$ an, so ist, wenn man zur Abkürzung

$$
\begin{gathered}
l \cdot\left(1+2 r \cos \alpha+r^{2}\right)^{\frac{1}{2}}=k \quad \text { und } \quad \operatorname{arc}\left(\operatorname{tang}=\frac{r \sin \alpha}{1+r \cos \alpha}\right)=h \text { setzt : } \\
(1+a+b i)^{m}=e^{p k-q h}[\cos (p h+q k)+i \sin (p h+q k)], \\
\left(\frac{1}{a+b i}\right)^{m}=e^{-p l(r)+q \alpha}[\cos (q l(r)+p \alpha)-i \sin (q l(r)+p \alpha)] \text { und } \\
\left(\frac{1}{a+b i}\right)^{m}(1+a+b i)^{m} \\
=e^{p(k-l(r))+q(a-h)}[\cos (p h-p \alpha+q k-q l(r))+i \sin (p h+q k-q l(r)-p \alpha)] .
\end{gathered}
$$

Die zweile Seite der Formel giebt:

$$
\begin{aligned}
1 & +\frac{D_{1} \cos E_{1}}{r}+\frac{D_{2} \cos E_{2}}{r^{2}}+\ldots \\
& -i\left[\frac{D_{1} \sin E_{1}}{r}+\frac{D_{2} \sin E_{2}}{r^{2}}+\ldots\right] .
\end{aligned}
$$

Crelle's Journal f. d. M. Bd. XXXIV. Heft 3. 
Demnach ist für $r>1$ :

2.

$\left\{\begin{aligned} 1+\frac{D_{1} \cos E_{1}}{r}+\frac{D_{2} \cos E_{2}}{r^{2}}+\ldots & =e^{\left.p\left(k-l_{1} r\right)\right)+q(\alpha-h)} \cos (p h-p \alpha+q k-q l(r)), \\ \frac{D_{1} \sin E_{1}}{r}+\frac{D_{2} \sin E_{2}}{r^{2}}+\ldots & =-e^{p(k+l(r))+q(\alpha-h)} \sin (p h-p \alpha+q k-q l(r)) .\end{aligned}\right.$

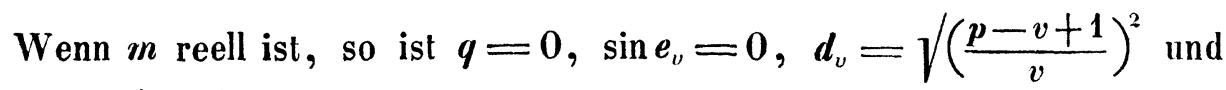
$\cos e_{l}=\frac{p-v+1}{v} \cdot \frac{1}{d_{v}}= \pm 1$. Übrigens erhält man leichter aus (1.) für $q=0$ :

3. $e^{p(k+l(r))}[\cos (p h-p \alpha)+i \sin (p h-p \alpha)]$

$$
\begin{aligned}
=1 & +\frac{p \cos \alpha}{r}+\frac{p \cdot(p-1)}{1 \cdot 2} \cdot \frac{\cos 2 \alpha}{r^{2}}+\ldots \\
& -i\left[\frac{p \sin \alpha}{r}+\frac{p \cdot(p-1)}{1 \cdot 2} \cdot \frac{\sin 2 \alpha}{r^{2}}+\ldots\right],
\end{aligned}
$$

woraus

$$
4 .\left\{\begin{array}{c}
1+\frac{p \cos \alpha}{r}+\frac{p \cdot(p-1)}{1 \cdot 2} \cdot \frac{\cos 2 \alpha}{r^{2}}+\ldots \\
e^{p l\left(1+2 r \cos \alpha+r^{2}\right)^{\frac{1}{2}}-p l(r)} \cos \left[p \operatorname{arc}\left(\operatorname{tang}=\frac{r \sin \alpha}{1+r \cos \alpha}\right)-p \alpha\right] \text { und } \\
\frac{p \cdot \sin \alpha}{r}+\frac{p \cdot(p-1)}{1 \cdot 2} \cdot \frac{\sin 2 \alpha}{r^{2}}+\cdots \\
=-e^{p l\left(1+2 r \cos \alpha+r^{2}\right)^{\frac{1}{2}}-p l(r)} \sin \left[p \operatorname{arc}\left(\operatorname{tang}=\frac{r \sin \alpha}{1+r \cos \alpha}\right)-p \alpha\right]
\end{array}\right.
$$

für $r$ positiv und $>1$ folgt.

Für $\alpha=\mathbf{0}$ findet sich hieraus

$$
\text { 5. } 1+\frac{p}{r}+\frac{p \cdot(p-1)}{1 \cdot 2 r^{2}}+\ldots=e^{p l\left(\frac{1+r}{r}\right)}=\left(\frac{1+r}{r}\right)^{p},
$$

für $r>1$.

Um die zweite der Formeln (4.) zu prüfen, setze man $p=1$ und $\alpha=\frac{1}{2} \pi$, wodurch sich ihre erste Seite auf $\frac{1}{r}$ reducirt. Die zweite Seite wird

$$
\begin{gathered}
-e^{l\left(1+r^{2}\right)^{\frac{1}{2}}-l(r)} \sin \left[(\operatorname{arctang}=r)-\frac{1}{2} \pi\right]=e^{l\left(\frac{1+r^{2}}{r^{2}}\right)^{\frac{1}{2}}} \cos \operatorname{arc}(\operatorname{tang}=r) \\
=\left(\frac{1+r^{2}}{r^{2}}\right)^{\frac{1}{2}} /\left(\frac{1}{1+r^{2}}\right)=\frac{1}{r},
\end{gathered}
$$

und stimmt mithin zusammen. 
Setzt man in den Formeln (4.) $\pi-\alpha$ statt $\alpha$, so erhält man

5.

$$
5 .\left\{\begin{array}{c}
1-\frac{p \cos \alpha}{r}+\frac{p \cdot(p-1)}{1 \cdot 2} \cdot \frac{\cos 2 \alpha}{r^{2}}-\ldots \\
\left(\frac{1-2 r \cos \alpha+r^{2}}{r^{2}}\right)^{\frac{1}{2} p} \cos \left[p \operatorname{arc}\left(\operatorname{tang}=\frac{r \sin \alpha}{1-r \cos \alpha}\right)+p \alpha-p \pi\right] \text { und } \\
\frac{p \sin \alpha}{r}-\frac{p \cdot(p-1)}{1 \cdot 2} \cdot \frac{\sin 2 \alpha}{r^{2}}+\ldots \\
=-\left(\frac{1-2 r \cos \alpha+r^{2}}{r^{2}}\right)^{\frac{1}{p} p} \sin \left[p \operatorname{arc}\left(\operatorname{tang}=\frac{r \sin \alpha}{1-r \cos \alpha}\right)+p \alpha-p \pi\right]
\end{array}\right.
$$

für $r>1$. Für $\alpha=0$ ergiebt sich hieraus:

$$
1-\frac{p}{r}+\frac{p \cdot(p-1)}{1 \cdot 2} \cdot \frac{1}{r^{2}}-\ldots=\left(\frac{1-2 r+r^{2}}{r^{2}}\right)^{\frac{1}{2} p} \cos (-p \pi) \text {. }
$$

Dies scheint beim ersten Anblick das Resultat zu sein; allein die zweite Formel würde

$$
\sin \boldsymbol{p} \pi=0
$$

geben; was aber offenbar in dieser Allgemeinheit nicht gilt. Es ist zu bemerken, dafs, da $1-r \cos \alpha=1-r$ wird, dies negativ ist, also dafs $\frac{r \sin \alpha}{1-r \cos \alpha}$ für $\alpha=0=-0$ wird und mithin $\operatorname{arc}\left(\operatorname{tang}=\frac{r \sin \alpha}{1-r \cos \alpha}\right)$ in diesem Falle $=\pi$ ist; dann erhält man

$$
\text { 6. } \quad 1-\frac{p}{r}+\frac{p \cdot(p-1)}{1 \cdot 2} \cdot \frac{1}{r^{2}}-\ldots=\left(\frac{r-1}{r}\right)^{p}
$$

für $r>1$.

Es zeigt sich hier an einem Beispiel, dafs eben so genau auf das Vorzeichen von $0 \mathrm{zu}$ achten sei, wie bei jeder andern Gröfse. Man hätte das nämliche Resultat übrigens auch finden können, wenn man $\alpha$ nur nach und nach der Null sich nähernd angenommen hätte.

\$. 12.

Die Reihe

$$
\text { 1. } x-\frac{x^{2}}{2}+\frac{x^{3}}{3}-\frac{x^{4}}{4}+\ldots
$$

wird für $x=a+b i=r(\cos \alpha+i \sin \alpha) \mathrm{zu}$

$$
\begin{aligned}
& \text { 2. } \quad r \cos \alpha-\frac{1}{2} r^{2} \cos 2 \alpha+\frac{1}{3} r^{3} \cos 3 \alpha-\ldots . \\
& +i\left[r \sin \alpha-\frac{1}{2} r^{2} \sin 2 \alpha+\frac{1}{3} r^{3} \sin 3 \alpha-\ldots\right] .
\end{aligned}
$$


Aber in (\$. 10. 1.) sahen wir, dafs

$$
\begin{aligned}
& r \cos \alpha-\frac{1}{2} r^{2} \cos 2 \alpha+\ldots=\frac{1}{2} l\left(1+2 r \cos \alpha+r^{2}\right) \text { und } \\
& r \sin \alpha-\frac{1}{2} r^{2} \sin 2 \alpha+\ldots=\operatorname{arc}\left(\operatorname{tang}=\frac{r \sin \alpha}{1+r \cos \alpha}\right)
\end{aligned}
$$

ist; mithin ist die Entwicklung (2.) oder (1.) gleich

$$
\text { 3. } \quad \begin{gathered}
\frac{1}{2} l\left(1+2 r \cos \alpha+r^{2}\right)+i \operatorname{arc}\left(\operatorname{tang}=\frac{r \sin \alpha}{1+r \cos \alpha}\right) \\
=\frac{1}{2} l\left[(1+a)^{2}+b^{2}\right]+i \operatorname{arc}\left(\operatorname{tang}=\frac{b}{1+a}\right) ;
\end{gathered}
$$

für den Fall nämlich, dafs (nach $\$$. 10. 1.) $r<1$, oder, wenn $r=1$, dafs nicht $\cos \alpha=-1$.

Die Gröfse (3.) ist aber der einfachste Werth von $l(1+a+b i)$, also ist die Reihe (1.) $=l(1+x)$, wenn man für $l(1+x)$ seinen einfachsten Werth setzt, vorausgesetzt, dafs $r<1$, oder $r=1$ und nicht zugleich $\cos \alpha=-1$ sei.

Da unter den angegebenen Bedingungen

$$
\text { 4. } x-\frac{x^{2}}{2}+\frac{x^{3}}{3}-\ldots=l(1+x)
$$

ist, so setze man $x=\frac{1}{a+b i}=\frac{1}{r}(\cos \alpha-i \sin \alpha)=\frac{1}{r}(\cos (-\alpha)+i \sin (-\alpha))$. Dies giebt

$$
\begin{aligned}
& \frac{1}{r} \cos \alpha+\frac{1}{2} \frac{1}{r^{2}} \cos 2 \alpha+\frac{1}{3} \frac{\cos 3 \alpha}{r^{3}}-\ldots-i\left(\frac{\sin \alpha}{r}-\frac{1}{2} \frac{\sin 2 \alpha}{r^{2}}+\frac{1}{3} \frac{\sin 3 \alpha}{r^{3}}-\ldots\right) \\
= & l(1+a+b i)-(l(r)+\alpha i)=\frac{1}{2} l\left(\frac{1+2 r \cos \alpha+r^{2}}{r^{2}}\right)+i\left(\operatorname{arctang}=\frac{r \sin \alpha}{1+r \cos \alpha}+\alpha\right),
\end{aligned}
$$
also ist für $r>1$ :

$$
\text { 5. }\left\{\begin{array}{l}
\frac{\cos \alpha}{r}-\frac{1}{2} \frac{\cos 2 \alpha}{r^{2}}+\frac{1}{3} \frac{\cos 3 \alpha}{r^{3}}-\ldots=\frac{1}{2} l\left(\frac{1+2 r \cos \alpha+r^{2}}{r^{2}}\right) \text { und } \\
\frac{\sin \alpha}{r}-\frac{1}{2} \frac{\sin 2 \alpha}{r^{2}}+\frac{1}{3} \frac{\sin 3 \alpha}{r^{3}}-\ldots=-\left[\operatorname{arc}\left(\operatorname{tang}=\frac{r \sin \alpha}{1+r \cos \alpha}-\alpha\right] .\right.
\end{array}\right.
$$

Diese Gleichungen hätten übrigens auch aus den Formeln (4. \$. 11.) hergeleitet werden können.

. Setzt man in diesen Gleichungen $\pi-\alpha$ statt $\alpha$, so erhält man:

6. $\left\{\begin{array}{l}\frac{\cos \alpha}{r}+\frac{1}{2} \frac{\cos 2 \alpha}{r^{2}}+\frac{1}{3} \frac{\cos 3 \alpha}{r^{3}}+\ldots .=-\frac{1}{2} l\left(\frac{1-2 r \cos \alpha+r^{2}}{r^{2}}\right), \\ \frac{\sin \alpha}{r}+\frac{1}{2} \frac{\sin 2 \alpha}{r^{2}}+\frac{1}{3} \frac{\sin 3 \alpha}{r^{3}}+\ldots=-\left[\operatorname{arc}\left(\operatorname{tang}=\frac{r \sin \alpha}{1-r \cos \alpha}\right)+\alpha-\pi\right],\end{array}\right.$ für $r>1$. 
§. 13.

Es sei die ins Unendliche fortgehende Reihe

1. $1+(a+b i) l \cdot(\alpha+\beta i)+\frac{(a+b i)^{2}(l \cdot(\alpha+\beta i))^{2}}{2 !}+\frac{(a+b i)^{3}(l \cdot(\alpha+b i))^{3}}{3 !}+\ldots$

zu summiren, wo der Gröfse $l \cdot(\alpha+\beta i)$ ihr einfachster Werth beigelegt wird.

Es sei $\alpha+\beta i=r(\cos \varphi+i \sin \varphi)$, so ist $l \cdot(\alpha+\beta i)=l(r)+\varphi i$, also $(\boldsymbol{a}+\boldsymbol{b i}) \boldsymbol{l} \cdot(\boldsymbol{\alpha}+\beta \boldsymbol{i})=\boldsymbol{a} \boldsymbol{l}(\boldsymbol{r})-\boldsymbol{b} \varphi+(\boldsymbol{b l}(\boldsymbol{r})+\boldsymbol{a} \varphi) \boldsymbol{i}$.

Vergleicht man dies mit dem in (\$.4.) Gesagten, so findet man, dafs die Reihe (1.), was auch $a, b, \alpha, \beta$ sein mögen,

2. $e^{a l(r)-b \varphi}[\cos (b l(r)+a \varphi)+i \sin (b l(r)+a \varphi)]$

zur Summe habe. Diese Gröfse ist aber der einfachste Werth von

3. $(\alpha+\beta i)^{a+b i}$,

so dafs also $(\alpha+\beta i)^{a+b i}$ die Summe des Ausdrucks (1.) ist.

§. 14.

Man betrachte die Reihe

$$
\text { 1. } 1-\frac{x^{2}}{2 !}+\frac{x^{4}}{4 !}-\frac{x^{6}}{6 !}+\ldots
$$

für $\boldsymbol{x}=\boldsymbol{a}+\boldsymbol{b i}=\boldsymbol{r}(\cos \varphi+-i \sin \varphi)$.

Der Ausdruck (1.) wird für $x=r(\cos \varphi+i \sin \varphi)$ :

2. $\quad 1-\frac{r^{2} \cos 2 \varphi}{2 !}+\frac{r^{4} \cos 4 \varphi}{4 !}-\ldots+i\left(-\frac{r^{2} \sin 2 \varphi}{2 !}+\frac{r^{4}}{4 !} \sin 4 \varphi-\ldots\right)$;

eine Reihe, die nach ( $($. 4.) immer convergent ist.

Setzt man in der ersten Reihe (2. \$. 5.) $\frac{1}{2} \pi-\varphi$ statt $\psi$, so erhält man $1-\frac{r^{2} \cos 2 \varphi}{2 !}+\frac{r^{4}}{4 !} \cos 4 \varphi-\ldots=\frac{1}{2}\left(e^{r \sin \varphi}+e^{-r \sin \varphi}\right) \cos (r \cos \varphi)$.

Setzt man auch in der zweiten Reihe (3. \$.5.) $\frac{1}{2} \pi-\varphi$ statt $\varphi$, so erhält man

$$
\frac{r^{2}}{2 !} \sin 2 \varphi-\frac{r^{4}}{4 !} \sin 4 \varphi+\ldots=\frac{1}{2}\left(e^{r \sin \varphi}-e^{-r \sin \varphi}\right) \sin (r \cos \varphi) .
$$

Die vorgelegte Reihe (2.) oder (1.) hat also zur Summe:

3. $\frac{1}{2}\left(e^{r \sin \varphi}+e^{-r \sin \varphi}\right) \cos (r \cos \varphi)-\frac{1}{2} i\left(e^{r \sin \varphi}-e^{-r \sin \varphi}\right) \sin (r \cos \varphi)$

$$
=\frac{1}{2}\left(e^{b}+e^{-b}\right) \cos a-\frac{1}{2} i\left(e^{b}-e^{-b}\right) \sin a=\cos (a+b i) \text {. }
$$

Es ist auch
4. $1-\frac{r^{2}}{2 !} \cos 2 \varphi+\frac{r^{4} \cos 4 \varphi}{4 !}-\ldots+i\left(\frac{r^{2} \sin 2 \varphi}{r !}-\frac{r^{4} \sin 4 \varphi}{4 !}+\ldots\right)$
$=\frac{1}{2}\left(e^{-r \sin \varphi}+e^{r \sin \varphi} \cos (r \cos \varphi)-\frac{1}{2} i\left(e^{-r \sin \varphi}-e^{r \sin \varphi}\right) \sin (r \cos \varphi)\right.$. 
\$. 15.

Auf gleiche Weise findet sich die Summe der immer convergenten Reihe

$$
\text { 1. } x-\frac{x^{3}}{3 !}+\frac{x^{5}}{5 !}+\frac{x^{7}}{7 !}+\ldots
$$

für $x=(a+b i)=r(\cos \varphi+i \sin \varphi)$.

Diese Reihe (1.) ist nämlich

2. $r \cos \varphi-\frac{r^{3} \cos 3 \varphi}{3 !}+\frac{r^{5} \cos 5 \varphi}{5 !}-\ldots+i\left(r \sin \varphi-\frac{r^{3} \sin 3 \varphi}{3 !}+\ldots\right)$.

Setzt man in der zweiten Gleichung (2. \$. 5.) $\frac{1}{2} \pi-\varphi$ statt $\varphi$, so erhält man

$$
r \cos \varphi-\frac{r^{3} \cos 3 \varphi}{3 !}+\frac{r^{5} \cos 5 \varphi}{5 !}-\ldots=\frac{1}{2}\left(e^{r \sin \varphi}+e^{-r \sin \varphi}\right) \sin (r \cos \varphi) \text {. }
$$

Setzt man eben so in der ersten Gleichung (3. \$. 4.) $\frac{1}{2} \pi-\varphi$ statt $\varphi$, so erhält man

$$
r \sin \varphi-\frac{r^{3} \sin 3 \varphi}{3 !}+\frac{r^{5} \sin 5 \varphi}{5 !}-\ldots=\frac{1}{2}\left(e^{r \sin \varphi}-e^{-r \sin \varphi}\right) \cos (r \cos \varphi) .
$$

Die Summe der Reihe (1.) oder (2.) ist also immer:

$$
\begin{gathered}
\quad \frac{1}{2}\left(\boldsymbol{e}^{r \sin \varphi}+\boldsymbol{e}^{-r \sin \varphi}\right) \sin (\boldsymbol{r} \cos \varphi)+\frac{1}{2} i\left(e^{r \sin \varphi}-\boldsymbol{e}^{-r \sin \varphi}\right) \cos (\boldsymbol{r} \cos \varphi) \\
=\frac{1}{2}\left(\boldsymbol{e}^{b}+\boldsymbol{e}^{-b}\right) \sin \boldsymbol{a}+\frac{1}{2} i\left(\boldsymbol{e}^{b}+\boldsymbol{e}^{-b}\right) \cos \boldsymbol{a}=\sin (\boldsymbol{a}+\boldsymbol{b} \boldsymbol{i}) .
\end{gathered}
$$

\$. 16 .

Es sei die Reihe

$$
\text { 1. } \frac{x}{1}+\frac{1}{2} \frac{x^{3}}{3}+\frac{1 \cdot 3}{2 \cdot 4} \cdot \frac{x^{5}}{5}+\frac{1 \cdot 3 \cdot 5}{2 \cdot 4 \cdot 6} \cdot \frac{x^{7}}{7}+\ldots
$$

für $x=\alpha+\beta i$ zu summiren. Es sei $\alpha+\beta i=r(\cos \varphi+i \sin \varphi)$, so ist bekanntlich nach (\$. 9.)

$$
\left(1-x^{2}\right)^{-\frac{1}{2}}=1+\frac{1}{2} x^{2}+\frac{1 \cdot 3}{2 \cdot 4} \cdot x^{4}+\frac{1 \cdot 3 \cdot 5}{2 \cdot 4 \cdot 6} \cdot x^{6}+\ldots,
$$

vorausgesetzt, dafs $r^{2}<1$, oder, wenn $r^{2}=1$, dafs $\cos 2 \varphi$ nicht $=+1$ sei. Integrirt man auf beiden Seiten und bemerkt, dafs

ist, so findet sich

$$
\int\left(1-x^{2}\right)^{-\frac{1}{2}} d x=\operatorname{arc}(\sin =x)
$$

2. $\quad \operatorname{arc}(\sin =x)=x+\frac{1}{2} \frac{x^{3}}{3}+\frac{1 \cdot 3}{2 \cdot 4} \cdot \frac{x^{5}}{5}+\frac{1 \cdot 3 \cdot 5}{2 \cdot 4 \cdot 6} \cdot \frac{x^{7}}{7}+\ldots$,

so dafs die Summe der Reihe (1.) gleich $\operatorname{arc}(\sin =x)$ ist, wenn $r<1$, oder, für den Fall, dafs $r=1$ und nicht $\cos 2 \varphi=+1$ ist. 
Für $\alpha+\beta i=r(\cos \varphi+i \sin \varphi)$ wird die Reihe (1.)

$$
\begin{gathered}
r \cos \varphi+\frac{1}{2} \cdot \frac{r^{3}}{3} \cos 3 \varphi+\frac{1 \cdot 3}{2 \cdot 4} \cos 5 \varphi \cdot \frac{r^{5}}{5}+\ldots . \\
+i\left(r \cos \varphi+\frac{1}{2} \cdot \frac{r^{3}}{3} \sin 3 \varphi+\frac{1 \cdot 3}{2 \cdot 4} \cdot \frac{r^{5}}{5} \sin 5 \varphi+\ldots\right) .
\end{gathered}
$$

Ferner ist bekanntlich:

$\operatorname{arc}(\sin =r(\cos \varphi+i \sin \varphi))=\operatorname{arc}\left(\sin =\frac{r \cos \varphi}{\left(\frac{1}{2} \sqrt{ }\left(1-2 r^{2} \cos 2 \varphi+r^{4}\right)+\frac{1}{2}\left(1+r^{2}\right)\right)^{\frac{1}{2}}}\right)$

$+i l\left[\left(\frac{1}{2} \sqrt{ }\left(1-2 r^{2} \cos 2 \varphi+r^{4}\right)+\frac{1}{2}\left(1+r^{2}\right)^{\frac{1}{2}}\right)+\frac{\sin \varphi}{\sqrt{ }\left(\sin ^{2} \varphi\right)}\left(\frac{1}{2} /\left(1-2 r^{2} \cos 2 \varphi+r^{4}\right)-\frac{1}{2}\left(1-r^{2}\right)\right)^{\frac{1}{2}}\right]$,

wo $\frac{1}{2} \sqrt{ }\left(1-2 r^{2} \cos 2 \varphi+r^{4}\right)$ immer positiv zu nehmen ist.

Also ist

$3 .\left\{\begin{array}{c}r \cos \varphi+\frac{1}{2} \cdot \frac{r^{3}}{3} \cos 3 \varphi+\frac{1 \cdot 3}{2 \cdot 4} \cdot \frac{r^{5}}{5} \cos 5 \varphi+\ldots \\ =\operatorname{arc}\left(\sin =\frac{r \cos \varphi}{\left(\frac{1}{2} \sqrt{ }\left(1-2 r^{2} \cos 2 \varphi+r^{4}\right)+\frac{1}{2}\left(1+r^{2}\right)\right)^{\frac{1}{2}}}\right) \text { und } \\ r \sin \varphi+\frac{1}{2} \cdot \frac{r^{3}}{3} \sin 3 \varphi+\frac{1 \cdot 3}{2 \cdot 4} \cdot \frac{r^{5}}{5} \sin 5 \varphi+\ldots \\ =l\left\{\left[\frac{1}{2} \gamma\left(1-2 r^{2} \cos 2 \varphi+r^{4}\right)+\frac{1}{2}\left(1+r^{2}\right)\right]^{\frac{1}{3}}+\frac{\sin \varphi}{\sqrt{\left(\sin ^{2} \varphi\right)}}\left[\frac{1}{2} \gamma\left(1-2 r^{2} \cos 2 \varphi+r^{4}\right)-\frac{1}{2}\left(1-r^{2}\right)\right]^{\frac{1}{1}}\right\},\end{array}\right.$ für $r<1$ und positiv; eben so für $r=1$, wenn nicht $\cos 2 \varphi=1$ ist. Für $\varphi=0$ erhält man

$$
\text { 4. } \quad r+\frac{1}{2} \cdot \frac{r^{3}}{3}+\frac{1 \cdot 3}{2 \cdot 4} \cdot \frac{r^{5}}{5}+\ldots=\operatorname{arc}(\sin =r)
$$

für jedes positive $r$, welches kleiner ist als 1 ; aber nicht für $r=1$.

Setzt man in den Formeln (3.) $\frac{1}{2} \pi-\varphi$ statt $\varphi$, so erhält man

$$
5 .\left\{\begin{array}{c}
r \sin \varphi-\frac{1}{2} \cdot \frac{r^{3}}{3} \sin 3 \varphi+\frac{1 \cdot 3}{2 \cdot 4} \cdot \frac{r^{5}}{5} \sin 5 \varphi-\ldots \\
=\operatorname{arc}\left(\sin =\frac{r \sin \varphi}{\left(\frac{1}{2} \sqrt{ }\left(1+2 r^{2} \cos 2 \varphi+r^{4}\right)+\frac{1}{2}\left(1+r^{2}\right)\right)^{\frac{1}{2}}}\right) \text { und } \\
r \cos \varphi-\frac{1}{2} \cdot \frac{r^{3}}{3} \cos 3 \varphi+\frac{1 \cdot 3}{2 \cdot 4} \cdot \frac{r^{5}}{5} \cos 5 \varphi-\ldots \\
=l\left\{\left[\frac{1}{2} \gamma\left(1+2 r^{2} \cos 2 \varphi+r^{4}\right)+\frac{1}{2}\left(1+r^{2}\right)\right]^{\frac{1}{2}}+\frac{\cos \varphi}{\sqrt{ }\left(\cos ^{2} \varphi\right)}\left[\frac{1}{2} \sqrt{ }\left(1+2 r^{2} \cos 2 \varphi+r^{+}\right)-\frac{1}{2}\left(1-r^{2}\right)\right]^{\frac{1}{2}}\right\}
\end{array}\right.
$$

5.

für $r>0$ und $<1$, so wie auch für $r=1$, wenn nicht $\cos 2 \varphi=-1$ ist. 


\section{\$. 17 .}

Es sei endlich die Reihe

$$
\text { 1. } \frac{x}{1}-\frac{x^{3}}{3}+\frac{x^{5}}{5}-\frac{x^{7}}{7}+\ldots
$$

für $x=\alpha+\beta i$ zu summiren.

Es ist bekanntlich, wenn $x=\alpha+\beta i=r(\cos \varphi+i \sin \varphi)$, nach (\$. 12.):

$$
\begin{aligned}
l(1+x i)=l(1+\alpha i-\beta)= & x i-\frac{(x i)^{2}}{2}+\frac{(x i)^{3}}{3}-\frac{(x i)^{4}}{4}+\ldots \\
= & i\left(x-\frac{x^{3}}{3}+\frac{x^{5}}{5}-\ldots\right) \\
& +\frac{x^{2}}{2}-\frac{x^{4}}{4} \ldots
\end{aligned}
$$

für $\alpha^{2}+\beta^{2}<1$, und für $\alpha^{2}+\beta^{2}=1$, wenn dann nicht $\beta=+1$ ist. Ferner ist nach demselben Paragraphen:

$$
l(1-x i)=l(1-\alpha i+\beta)=i\left(-x+\frac{x^{3}}{3}-\frac{x^{5}}{5}+\ldots\right)+\frac{x^{2}}{2}-\frac{x^{4}}{4}+\frac{x^{6}}{6}-\ldots
$$

wenn $\alpha^{2}+\beta^{2}<1$, so wie auch wenn $\alpha^{2}+\beta^{2}=1$ ist; nur darf dann nicht $\beta=-1$ sein.

Also ist $l(1+x i)-l(1-x i)=$

$$
2 i\left(x-\frac{x^{3}}{3}+\frac{x^{5}}{5}-\ldots\right)
$$

Die vorgelegte Reihe ist demnach $=\frac{l(1+x i)-l(1-x i)}{2 i}$, für den Fall, dafs $r<1$, so wie für den Fall, wenn $r=1$ ist; nur darf dann nicht $\sin ^{2} \varphi=1$ sein. Diese Bedingungen reduciren sich auf das Folgende. Die Reihe hat die angegebene Summe für $\alpha^{2}+\beta^{2}<1$, und wenn $\alpha^{2}+\beta^{2}=1$ ist; nur darf in letzterem Falle $\alpha$ nicht Null sein. Aber es ist bekanntlich:

$$
\begin{gathered}
\frac{l(1+x i)-l(1-x i)}{2 i}=\frac{l(1-\beta+\alpha i)-l(1+\beta-\alpha i)}{2 i} \\
=\frac{1}{2}\left[\operatorname{arc}\left(\operatorname{tang}=\frac{\alpha}{1-\beta}\right)+\operatorname{arc}\left(\operatorname{tang}=\frac{\alpha}{1+\beta}\right)+\frac{1}{4} i \cdot l \cdot \frac{(1+\beta)^{2}+\alpha^{2}}{(1-\beta)^{2}+\alpha^{2}}\right. \\
=\frac{1}{2} \operatorname{arc}\left(\operatorname{tang}=\frac{2 \alpha}{1-\beta^{2}-\alpha^{2}}\right)+\frac{1}{4} i \cdot l \cdot\left(\frac{(1+\beta)^{2}+\alpha^{2}}{(1-\beta)^{2}+\alpha^{2}}\right)=\operatorname{arc}(\operatorname{tang}=\alpha+\beta i) .
\end{gathered}
$$

Demnach ist die Reihe gleich $\operatorname{arc}(\operatorname{tang}=x)$, wenn nemlich $r<1$, und wenn $r=1$, aber nicht zugleich $\sin ^{2} \varphi=1$ ist.

$\mathrm{Da} \alpha=r \cos \varphi, \beta=r \sin \varphi$ ist, so ist $\frac{2 \alpha}{1-\beta^{2}-\alpha^{2}}=\frac{2 r \cos \varphi}{1-r^{2}}$ und $\frac{(1+\beta)^{2}+\alpha^{2}}{(1-\beta)^{2}+\alpha^{2}}=\frac{1+2 r \sin \varphi+r^{2}}{1-2 r \sin \varphi+r^{2}}$; also ist offenbar: 
2. $\left\{\begin{array}{l}r \cos \varphi-\frac{r^{3}}{3} \cos 3 \varphi+\frac{r^{3} \cos 5 \varphi}{5}-\ldots=\frac{1}{2} \operatorname{arc}\left(\operatorname{lang}=\frac{2 r \cos \varphi}{1-r^{2}}\right) \\ r \sin \varphi-\frac{r^{3}}{3} \sin 3 \varphi+\frac{r^{5} \sin 5 \varphi}{5}-\ldots=\frac{1}{4} l\left(\frac{1+2 r \sin \varphi+r^{2}}{1-2 r \sin \varphi+r^{2}}\right)\end{array}\right.$

für $r<1$ und $r=1$, wenn nicht $\sin ^{2} \varphi=1$ ist.

Setzt man $\frac{1}{2} \pi-\varphi$ statt $\varphi$, so findet sich:

3. $\left\{\begin{array}{l}r \sin \varphi+\frac{r^{3}}{3} \sin 3 \varphi+\frac{r^{5} \sin 5 \varphi}{5}+\ldots=\frac{1}{2} \operatorname{arc}\left(\operatorname{tang}=\frac{2 r \sin \varphi}{1-r^{2}}\right), \\ r \cos \varphi+\frac{r^{3}}{3} \cos 3 \varphi+\frac{r^{5} \cos 5 \varphi}{5}+\ldots=\frac{1}{4} l\left(\frac{1+2 r \cos \varphi+r^{2}}{1-2 r \cos \varphi+r^{2}}\right),\end{array}\right.$

für $r<1$ und $r=1$, wenn nicht $\cos ^{2} \varphi=1$ ist.

Es läfst also die Gröfse $\operatorname{arc}(\operatorname{tang}= \pm i)$ nicht durch eine Reihe sich darstellen; wie sie sich bekanntlich auch nicht durch einen geschlossenen Ausdruck darstellen läfst.

Für $r=1$ erhält man

$$
\text { 4. }\left\{\begin{array}{l}
\cos \varphi-\frac{1}{3} \cos 3 \varphi+\frac{1}{5} \cos 5 \varphi-\ldots=\frac{1}{4} \pi \\
\sin \varphi-\frac{1}{3} \sin 3 \varphi+\frac{1}{5} \sin 5 \varphi-\ldots=\frac{1}{4} l\left(\frac{1+\sin \varphi}{1-\sin \varphi}\right)
\end{array}\right.
$$

was von $\varphi=0$ bis $\varphi=\frac{1}{2} \pi$, und im Allgemeinen für positive Werthe von $\cos \varphi$ gilt, aufser wenn $\cos \varphi=0$ ist. Die zweite dieser Gleichungen gilt auch für negative Werthe von $\cos \varphi$; für solche aber geht die erste in

5. $\cos \varphi-\frac{1}{3} \cos 3 \varphi+\frac{1}{5} \cos 5 \varphi-\ldots=-\frac{1}{4} \pi$

über, aufser für $\cos \varphi=0$. Die Gleichungen (3.) geben:

6. $\sin \varphi+\frac{1}{3} \sin 3 \varphi+\frac{1}{5} \sin 5 \varphi+\ldots=\frac{1}{4} \pi$

für positive Werthe von $\sin \varphi$, aufser für $\sin \varphi=0$,

7. $\sin \varphi+\frac{1}{3} \sin 3 \varphi+\frac{1}{5} \sin 5 \varphi+\ldots=-\frac{1}{4} \pi$

für negative Werthe von $\sin \varphi$, aufser für $\sin \varphi=0$, und

8. $\cos \varphi+\frac{1}{3} \cos 3 \varphi+\frac{1}{5} \cos 5 \varphi+\ldots=\frac{1}{4} l\left(\frac{1+\cos \varphi}{1-\cos \varphi}\right)=\frac{1}{2} l \cdot(\operatorname{cotan} \varphi)$, aufser für $\sin \varphi=0$.

Es lassen sich einige dieser Reihen durch Inlegration leicht zu Grundformeln einer neuen Reihe von Formeln benutzen. Ihre Aufstellung ist sehr einfach.

\section{§. 18.}

Im Vorstehenden sind die in der Analysis gebräuchlichsten Functionen in Reihen entwickelt, oder vielmehr die Reiben durch geschlossene Ausdrücke Crelle's Journal f. d. M. Bd. XXXIv. Heft 3. 
summirt worden, und es sind dabei die Grenzen angegeben, innerhalb welcher die gefundenen Resultate Geltung haben. Es lassen sich noch mancherlei Folgerungen aus den gefundenen Resultaten ziehen; wir haben sie nicht hergesetzt, weil es sich nur darum handelte, die so oft und auf so mancherlei Art behandelten Reihen streng zu untersuchen, um in jedem Falle mit mathematischer Gewilsheit entscheiden zu können, ob eine bestimmte Reihe anwendbar sei, oder nicht. Es ist nachgewiesen worden, dafs die untersuchten Reihen, wenigstens ihrer gröfsern Zahl nach, nur innerhalb gewisser Grenzen Summen haben, und dafs aufserhalb jener Grenzen die Reihen ohne Bedeutung und ohne Bestand, also nicht anwendbar sind.

Zum Schlusse möge noch Einiges in Bezug auf die obigen Differenziirungen und Integrirungen bemerkt werden.

Ist $\varepsilon$ eine unendliche kleine Gröfse, so sagen wir, die unendlich kleine Gröfse $\boldsymbol{k}$ sei unendlich klein von der Ordnung $\boldsymbol{r}$, wenn das Verhältnifs

$$
\frac{k}{\varepsilon^{n}}
$$

unendlich wird für $n>r$, Null für $n<\boldsymbol{r}$ und endlich (auch Null, nicht aber unendlich) für $\boldsymbol{n}=\boldsymbol{r}$; alles in Bezug auf $\varepsilon$. Ferner nennen wir die Function $f(x)$ continuirlich innerhalb bestimmter Grenzen von $x$, wenn $f(x+\varepsilon)-f(x)$ innerhalb jener Grenzen eine unendlich kleine Gröfse von gleicher oder höherer Ordnung ist, als $\varepsilon$. Das Verhältnifs $\frac{f(x+\varepsilon)-f(x)}{\varepsilon}$ ist also in jedem Falle endlich; und umgekehrt, wenn dies Verhältnifs endlich ist, so ist $f(x)$ continuirlich. Diese Gegenseiligkeit hat gemacht, dafs man jenes Verhältnifs besonders heraushebt und durch $\frac{d f(x)}{d x}$ (den Differentialquotienten von $f(x)$ ) bezeichnet. So lange derselbe also endlich ist, bleibt $f(x)$ continuirlich.

Im Vorstehenden sind die Werthe der unendlichen Reihen für endliche Werthe der darin vorkommenden Gröfsen bestimmt worden; auf die Änderungen, welche diese Reihen durch unendlich kleine Änderungen der Gröfsen erleiden, und auf den Gang dieser Änderungen, hat man nicht geachtet. Es ist daher denkbar, dafs der geschlossene Ausdruck, der die Summe der Reihe darstellt, continuirlich im oben angegebenen Sinne sei, während die Reihe selbst nicht continuirlich ist, obwohl für jeden bestimmten Werth die beiden Ausdrücke zusammenfallen. Es wird also, wenn man auf beiden Seiten der entwickelten Formeln differenziirt, ohne vorhergehende Untersuchung nicht 
angenommen werden dürfen, dafs die beiden gefundenen Differentialquotienten gleich seien, indem es sich ereignen kann, dafs die vorgelegte summirle Reihe nicht continuirlich (im obigen Sinne) ist und deshalb ihr Differenzialquotient gar nicht Statt findet. Integrirt man aber beiderseits, so sind die Integrale ohne Weiteres gleich zu setzen; denn die erhaltene Reihe ist sogar continuirlich (im aufgeführten Sinne), weil ihr Differentialquotient endlich ist; und zwar innerhalb der Grenzen, in welchen die Reihe selbst summirt wird.

So z. B. geben die Reihen (6. und 7. \$. 17.), wenn man nach $q$ differenziirt,

$$
\cos \varphi+\cos 3 \varphi+\cos 5 \varphi+\cos 7 \varphi+\ldots=0 \text { : }
$$

ein Resultat, welches offenbar unrichtig ist. Die Reihe

$$
\cos \varphi+\cos 3 \varphi+\cos 5 \varphi+\ldots
$$

ist divergent, mithin ist

$$
\sin \varphi+\frac{1}{3} \sin 3 \varphi+\frac{1}{5} \sin 5 \varphi+\ldots
$$

nicht continuirlich. Ähnliche Resultate finden sich aus den andern Formeln. Dagegen giebt die Integration der Formel (6.):

$$
\cos \varphi+\frac{1}{3^{2}} \cos 3 \varphi+\frac{1}{5^{2}} \cos 5 \varphi+\ldots=-\frac{1}{4}(\pi \varphi)+C,
$$

für positive Werthe von $\sin \varphi$, aufser für $\sin \varphi=0$, also blofs von $\varphi=0$ bis $\varphi=\pi$. Für $\varphi-\frac{1}{2} \pi$ ist die erste Seite Null, also

$$
\begin{gathered}
C=\frac{1}{2} \pi^{2} \quad \text { und } \\
\cos \varphi+\frac{1}{3^{2}} \cos 3 \varphi+\frac{1}{5^{2}} \cos 5 \varphi+\ldots=\frac{1}{8} \pi^{2}-\frac{1}{4}(\pi \varphi) .
\end{gathered}
$$

Diese Bemerkungen, deren weitere Ausführung nicht hieher gehört, wcrden bei den Umbildungen durch Differenziirung und Integrirung maafsgebend sein; sie enthalten vielleicht die Auflösung der Frage, die Abel in dem von $\mathrm{O} / \mathrm{m}$ in seinem „Geist der math. Analysis" S. 3 angeführten Briefe gestellt hat.

Sinsheim bei Heidelberg im Mai 1845. 